\section{UCDNN}

LIBRARY
University of Connecticut OpenCommons@UConn

8-13-2018

\title{
Toward a Culturally Robust Measure to Assess Marital Relationships
}

Hyanghee Lee

University of Connecticut - Storrs, hyanghee.lee@uconn.edu

Follow this and additional works at: https://opencommons.uconn.edu/dissertations

\section{Recommended Citation}

Lee, Hyanghee, "Toward a Culturally Robust Measure to Assess Marital Relationships" (2018). Doctoral Dissertations. 1890. https://opencommons.uconn.edu/dissertations/1890 
Toward a Culturally Robust Measure to Assess Marital Relationships

Hyanghee Lee, Ph.D.

University of Connecticut, 2018

The Marital Comparison Level Index (MCLI; Sabatelli, 1984), grounded in social exchange theory, was developed to measure marital complaints by asking respondents to contrast their marital experiences with their marital expectations. Translated versions of the MCLI have been used in non-Western cultures such as in Korea and China (e.g., Chan \& Rudowicz, 2002; Yang, 2004). However, since each individual's standards and expectations for evaluating relationships are culturally influenced, it follows that measures reflecting Western cultural values may not be reliably applied to different cultural settings. Therefore, the goal for this study was to revise the measure in a culturally relevant way to be used to study Asian couples, with a particular emphasis on Korean couples.

Guided by an ecological/exchange framework (Sabatelli, Lee, \& Ripoll-Núñez, 2018), the culture-specific aspects (e.g., intergenerational exchanges) of marital relationships were included along with contemporary aspects of marriages (e.g., technology use, work-torelationship spillover) in the revisions of the MCLI. Using the sample of Korean and American married individuals ( $\mathrm{N}=676)$, measurement invariance testing was conducted to assess whether the measure performs in the same way across two cultures. Results showed that there was a second-order factor, which is marital quality, that underlies the five firstorder factors (i.e., emotional intimacy, sexual intimacy, marital conflicts, intergenerational relationships, and complaints about partner's lifestyle). The identified second-order factor structure showed an adequate level of measurement invariance, indicating the potential for 
Hyanghee Lee - University of Connecticut, 2018

explaining cross-cultural relevance of the marital construct. Taken together, the present study serves as an impetus for international scholarship that could promote cultural and racial diversity in relationship research. 
Toward a Culturally Robust Measure to Assess Marital Relationships

Hyanghee Lee

B.A., Seoul National University, 2010

M.A., Seoul National University, 2012

\author{
A Dissertation \\ Submitted in Partial Fulfillment of the \\ Requirements for the Degree of \\ Doctor of Philosophy \\ at the \\ University of Connecticut
}


Copyright by

Hyanghee Lee

2018 
APPROVAL PAGE

Doctor of Philosophy Dissertation

Toward a Culturally Robust Measure to Assess Marital Relationships

Presented by

Hyanghee Lee, B.A., M.A.

Major Advisor

Ronald M. Sabatelli

Associate Advisor

Noel Card

Associate Advisor

Kari Adamsons

University of Connecticut

2018 
Table of Contents

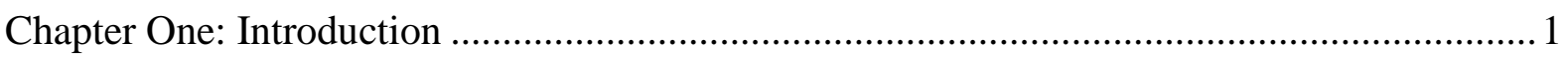

Chapter Two: Review of Relevant Literature .................................................................... 5

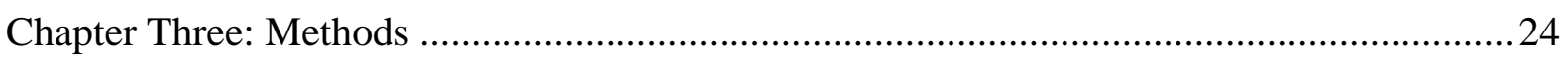

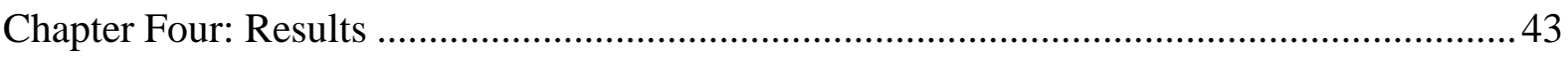

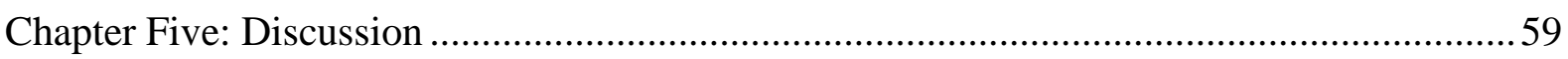

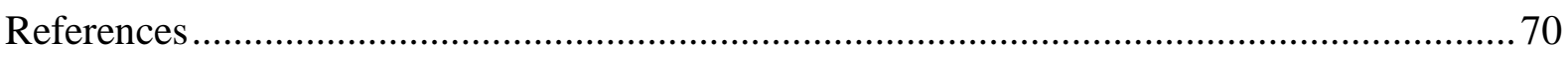

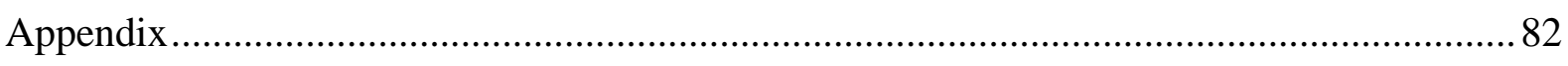




\section{CHAPTER ONE: INTRODUCTION}

In recent years, there has been noticeable growth in the international scholarship focusing on marital and family issues. In many instances, international scholars have adapted measures created by U.S. scholars for use in their studies. The use of these adapted measures is grounded in the unstated premise that measures created by U.S. scholars are culturally robust, meaning that in spite of differences in cultural value orientations, customs, practices, and/or policies, the conceptualization and operationalization of a construct are invariant. For example, the measurement of relationship qualities such as marital quality, marital satisfaction, and marital adjustment has been extensively studied in the West and marital researchers from different parts of the world have applied these marital constructs and measures to their respective cultures, including Asian countries. It has become common to use a translated version of marital assessment tools without consideration to the possibility that the construct that the scale is designed to assess may need to be re-conceptualized or reoperationalized in order to be more sensitive to cultural values and norms (Yu, 2015).

Given the fact that families are embedded in a socio-cultural and historical context, it is critical to take into account specific cultural norms and social expectations that filter into the experiences of marriage. It is apparent that decades of attention to research on the psychometric properties of Western scales has obscured the importance of the need to capture the cultural specifics of marital constructs. In this regard, marital researchers who conduct research on Asian families have begun to incorporate cultural specificity into the measurement by adding culture-specific items to the original scales (e.g., Chan \& Rudowicz, 2002; Madathil \& Benshoff, 2008; Yang, 2004). However, many of these attempts have not been accompanied by rationales addressing the human universals and/or culture-specific aspects of marital relationships. 
Based on social exchange framework and, particularly, the theory of Thibaut and Kelley (1959), the Marital Comparison Level Index (MCLI; Sabatelli, 1984) was developed to measure marital complaints within different domains of a relationship by contrasting between marital experiences and marital expectations. Specifically, the items of the MCLI tapped into important rewards and costs associated with marital experiences. These items were scaled to allow respondents to indicate how their experiences compare with their baseline of expectations. In other words, respondents could evaluate their relationship outcomes as being either relatively worse than they expect, about what they expect, or better than they expect. Evidence for the construct validity of this measure was supported by the finding that complaints varied in predicted ways with each partners' experiences of equity, fairness, affection, and commitment within marriages (Sabatelli, 1984; Sabatelli \& BartleHaring, 2003).

Translated versions of the MCLI have been used in non-Western cultures such as China and Korea (e.g., Shek, Lam, Tsoi, \& Lam, 1993; Yang, 2004), indicating the universal appeal of the social exchange framework when applied to the understanding of how marriages are structured and experienced. However, since each individuals' standards and expectations for evaluating relationships are culturally influenced, it follows that measures based primarily on individualism in Western cultures may not be reliably applied to different cultural settings. In line with this, many of the attempts to modify the MCLI involved the addition of supposedly culture-specific items without providing a culturally grounded rationale or empirical/psychometric support for the addition of these items (e.g., Chan \& Rudowicz, 2002; Yang, 2004). Furthermore, it is important to point out the methodological limitations associated with the use of translated measures. Previous researchers who evaluated the psychometric properties of the MCLI within a different cultural context examined only the reliability or the factor structure of the measure in order to ensure the 
equivalence of the scale. For example, a validation study of the translated version of the MCLI reported acceptable levels of reliability and validity as a "one size fits all" solution to validate the measure in Korean and Chinese samples (Shek, Lam, Tsoi, \& Lam, 1993; Yang, 2004). These studies, however, lacked evidence for measurement invariance, which would suggest whether the measure performs in the same way across cultures (Byrne \& Campbell, 1999; Byrne \& van de Vijver, 2014; Kline, 2011). That is, equivalence testing of data gained from respondents in many cultures is needed in order to support the conclusion, from a statistical point of view, that a measure is cross-culturally relevant.

\section{Purpose of Study}

The primary goal of this study is to complete conceptual and psychometric revisions to the MCLI in a culturally appropriate way and test for evidence of the revised measure's cross-cultural relevance. That is, this study examines whether certain factors (e.g., intergenerational relationships) developed indigenously in Korean cultures are relevant to Western cultures through a series of statistical analyses (i.e., measurement invariance testing). In doing so, results of the study would have the potential for explaining either systematic cultural differences or the cross-cultural relevance of the marital construct, which in turn, contributes to the advancement of marital research that provides better understandings of family dynamics in different sociocultural contexts.

\section{Organization of the Dissertation}

In the next chapter, I review the relevant literature for developing a culturallysensitive measure for use with a Korean population. The review begins with an overview of an ecological/exchange framework that provides a rationale for focusing on contextual/cultural factors when applying the Western scale to different cultural contexts. 
This is followed by a literature review of culture-specific aspects of marital relationships that need to be included in the revised measure for Koreans through the lens of the ecological/exchange framework. Furthermore, given the fact that there is a need for the creation of more modern and psychometrically sound marital assessment tools in marital research, Chapter Two includes factors that need to be considered to measure contemporary families across cultures. In doing so, the refinement of the MCLI opens up the possibility of exploring the continuum between human universals and culturally specific aspects of marital relationships. This is followed by an overview of equivalence testing of data gained from respondents in different cultures, from a statistical point of view.

Chapter Three presents the methods used in this study, including the "cultural revision" process of the MCLI, recruitment strategies, data collection procedures, and descriptions of the included measures. This chapter also includes an explanation of the multiphasic structure of the data analysis.

Chapter Four includes a description of the results for each phase of the study. Details of quantitative cross-cultural comparisons of the results follow.

Chapter Five discusses the current study's findings, conclusions that can be drawn from these findings, and its limitations. Implications for the practical use of the measure in two cultural contexts and future research are then suggested. 


\section{CHAPTER TWO: REVIEW OF RELEVANT LITERATURE}

This chapter begins with a brief overview of the social exchange framework of how intimate relationships are structured and experienced. This theoretical perspective is expanded to the ecological/exchange framework to acknowledge how contextual factors influence marital exchanges. The ecological/exchange framework is presented to provide a rationale for the cultural revision of the MCLI. Using this framework, previous findings are organized around the contextual factors that have been found to influence the quality of marital relationships focusing on Korean couples, namely intergenerational exchanges of married individuals with their parents and parents-in-law. Furthermore, in order to reflect historical changes in marriage and family structure, this chapter includes a review of the literature on factors that need to be included in the revised measure for assessing contemporary intimate relationships (i.e., technology use, changing time allocation of couples, and work-to-relationship spillover). Lastly, this chapter provides an overview of the literature regarding the importance of testing the extent to which measurement properties generalize across cultures.

\section{Theoretical Perspective}

\section{Social Exchange Framework within Context: An Ecological/Exchange Framework}

Social exchange theories have been utilized to explain how intimate relationships are structured and experienced. The framework has been used to account for relationship formation and interpersonal attraction, mate selection, and relationship dissolution (McDonald, 1981). The basic assumption is that individuals are attracted to others when there are positive outcomes from the relationships (Sabatelli, 1999; Sabatelli \& Shehan, 1993). Specifically, Thibaut and Kelley (1959) used the concept of rewards and costs to explain social exchanges that occur in a relationship in ways that maximize rewards and minimize 
costs. Rewards are defined as the "pleasures, satisfactions, and gratifications the person enjoys" (Thibaut \& Kelley, 1959, p.12). Costs are any negative experiences that result from being involved in a relationship (Nye, 1979; Sabatelli, 1999). The rewards and costs are evaluated by comparing experiences to the comparison level (CL) - that is, by comparing experiences to what is realistically expected, thought to be deserved, and deemed critical or salient. However, these comparisons of outcomes to expectations or orientations do not, by themselves, explain why people stay in or leave either rewarding or unsatisfying relationships. To fill this gap, Thibaut and Kelley (1959) introduced the concept of comparison level for alternatives (CL alt), which refers to the lowest level of relationship outcomes a person can experience before taking available alternatives into consideration. People who experience a low CL and low CL alt in their current relationships tend to have a high level of dependence on that relationship, which can lead them to stay in a relationship even if it is unsatisfying. In other words, people leave the relationship only when they have better alternatives and think that the benefits of terminating the current relationship outweigh the costs of leaving (Thibaut \& Kelley, 1959). Dependence as it interacts with satisfaction or rewards, therefore, determines the likelihood that a relationship will be maintained.

Despite its focus on rewards, costs, and alternatives to explain what contributes to relationship satisfaction and relationship quality, a persistent criticism of the exchange framework is that it does not reflect the ways exchange relationships are experienced and structured by a constellation of social, economic, and political factors in a broader context. To address this issue, Sabatelli and Ripoll-Núñez (2003) developed an "ecological/exchange framework" to explain how ecological factors influence exchange relationships. They argued that cultural value orientations or macro-level factors (e.g., individualism vs. collectivism, egalitarianism vs. hierarchy) influence normative exchange orientations, which, in turn, leads to different structures and experiences of marital relationships in a given context. For 
example, Sabatelli and Ripoll-Núñez (2003) discussed how changes in educational opportunities for women and shifts in the perceptions of modern values with respect to gender equality, have resulted in changes in how intimate relationships are structured and experienced over the past fifty years. These social changes, from the exchange perspective, are reflected in both the expectations that men and women have for their relationships and the expectations they have for alternative relationships. As an example, they explained how the rapid increase in divorce in the late 20th century in Western cultures was accompanied by a confluence of factors that influenced women's CL and CLalt—namely, increases in access to education and employment along with the development and more widespread availability of reliable contraception. This perspective complements the social exchange framework based on Western cultures derived from individualistic assumptions by acknowledging how cultural value orientations and conventions filter into the ways in which exchange relationships are negotiated. More recently, Sabatelli, Lee, and Ripoll-Núñez (2018) expanded the ecological/exchange framework to more completely understand cultural, cohort, and socioeconomic variations that exist within interdependent exchange relationships. In other words, there is variability within individuals that are from the same culture, suggesting that we need to consider the possibility that a study population consists of multiple subgroups characterized by qualitatively and quantitatively distinct indicators (e.g., individual characteristics).

\section{The Macrosystem and the Structure and Experience of Intimate Relationships in Korea}

The following literature review on marital relationships in Korea was organized using the ecological/exchange framework (Sabatelli \& Ripoll-Núñez, 2003; Sabatelli, Lee, \& Ripoll-Núñez, 2018). This framework was applied to culture-specific aspects of intimate relationships in Korea to focus on ecological factors that influence intimate exchanges. 
Macro-level values. Within the ecological/exchange framework, cultural value orientations guide certain expectations about how marriages are structured, experienced, and evaluated in a given society. For example, collectivistic values in Korea are manifested in an emphasis on familism, which refers to a family-centered worldview (Rappa \& Tan, 2003). Familism in Korea is uniquely influenced by Confucian philosophy and places a strong emphasis on filial piety, intergenerational loyalties, and support of the patrilineal family over the matrilineal family $(\mathrm{Ok}, 2011)$. In traditional Confucian familism, it is expected that couples go predominantly to the husbands' family for support between generations, resulting in the wife's frequent contact with and obligation toward her in-laws. The gendered filial norms of Korean families show obligatory patterns in supporting parents-in-law financially and/or emotionally, which, in turn, potentially, leads to tensions and conflicts not only in the marital relationships, but also in intergenerational relationships (i.e., the relationship between a mother-in-law and a daughter-in-law). The cultural practices regarding intergenerational relationships in Korean families are also explained by a hierarchy based on gender and age. Sons are expected to provide primary support for their older parents, while married daughters are more likely to be emotional caregivers for their own parents and important caregivers (e.g., instrumental support) for their elderly parents-in-law (Kim, Zarit, Fingerman, \& Han, 2015).

Given the prominence of the patrilineal family system described above, Korean scholars have increasingly emphasized understanding the causal significance of the relationship with in-laws for marital relationships (Choi \& Han, 2011; Park \& Cho, 2010; Yoo, Cho, \& Kwon, 2008). For example, Yoo, Cho, and Kwon (2008) revealed the negative effects of family-of-origin experiences and conflicts with in-laws on marital satisfaction. Similarly, Park and Cho (2010) revealed the negative association between the relationship quality with partner and conflicts with their parents-in-law among married women. Given the 
gendered filial norms of Korean families, it is not surprising that Korean scholars have increasingly emphasized the role of in-law relationships in determining marital satisfaction and adjustment (Choi \& Han, 2011; Park \& Cho, 2010; Yoo, Cho, \& Kwon, 2008).

Such cultural value orientations have been reflected in the measures used to assess the perception of marital relationships focusing on Korean couples. For example, the Marital Satisfaction Inventory (Snyder, 1997) was modified by Kwon and Choi (1999) for Koreans by adding culture-specific items such as relationships with in-laws. Similarly, Chung (2004) included conflicts with in-laws to the constructs of marital satisfaction in her endeavor to develop a culturally-sensitive measure for use with Korean married individuals. Interestingly, however, little research has been done regarding how problematic relationships with parentsin-law influence adult children's marital quality in the U.S., probably because married couples in the United States are relatively autonomous from in-laws (Morr Serewicz, 2006). Further, the norm of equity between partners is more salient in intimate relationships, whereas couples go predominantly to the husbands' family for support between generations in Korea, which is also tied to cultural values in a given society. It implies that what is considered to be important in Asian cultures might not receive much attention in Western culture. Taken together, these findings highlight a need to consider cultural specificity in the measurement of marital constructs.

Time as an exchange context. Due to historical and social changes, cultural values and norms have shifted, with greater diversity in the expectations and standards that individuals hold regarding marital relationships over time. As proposed by the ecological/exchange framework, macro-level factors (i.e., cultural value orientations) influence individuals' normative exchange orientations, which show a greater variability within and between cohorts over time. For example, with the recent educational and 
economic growth in Korea, married couples' relationships with their parents may be somewhat different from traditional patterns of support given to older parents. A recent Korean study (Statistics Korea, 2016) about attitudes on the relationship with family of origin reveals more flexibility about intergenerational loyalties and intimate partnerships. An interesting point suggested in this study has to do with the variability found in cohort differences, with almost half (45\%) of young adults (30-39 years) reporting that their intimate partnership had priority, whereas middle-aged respondents (50-59 years) reported the opposite $-60 \%$ of them put their relationship with family of origin first (Statistics Korea, 2016). These results highlight the variations within cultures and the specific ways in which individuals within the same culture may or may not adopt the same value orientations and how these might shift over time.

Cultural norms, customs, and practices of social institutions. In the past thirty years, Korea has experienced significant demographic and economic changes, which contributed to a transformation in the patterns of family structure and relationships. Along with boasting the highest rate of college education in the world, the gender gap in educational attainment has significantly narrowed (OECD, 2017). Traditional male-headed households are on the decline due to the meteoric rise in female employment, which is consistent with increased opportunity for women in higher education (OECD, 2017). Also, with increased access to contraception and changing perceptions of childbearing, fertility rates have reached a record low of 1.08 children per woman in 2005 (OECD, 2018). These numbers reflect changes in the expectations of and dependence on marriages, showing how contextual forces shape and structure marital exchange relationships.

It is also important to consider how micro-level patterns and dynamics within marital exchange relationships have changed in response to social, cultural, and economic changes. 
One such example is the increasing divorce rates. According to Statistics Korea (2017), the Korean crude divorce rate (CDR: the number of divorces per 1,000 people) stood at 2.1 cases in 2016 after an upsurge to 3.5 cases in 2003. Although the CDR is lower in Korea than in the U.S., - 3.2 cases in 2016, ("Marriage and Divorce," n.d.) — the pattern is similar to that in other nations when modernization results in increased economic and educational opportunities for women. That is, as educational and economic opportunities for women have expanded and social norms and policies have changed to protect their rights, women are more likely to leave unsatisfying relationships than in the past. The increase in divorce may suggest that contextual forces that lessen the dependence of women on marriage play a major role in micro-level decisions in marriages. Also, it shows how historical factors impact divorce rates within countries over time. For example, relatively rapid shifts in marriage, family, and divorce patterns have occurred in Korea over the past 30 years. Korea now boasts the highest rate of college education in the world ( $70 \%$ of the Korean population holds a college degree) and historically low fertility rates (OECD, 2018). Similar to U.S. divorce trends, three interrelated factors-women's increased access to higher education, the ability to control fertility based on diffusion of contraceptive technology, and rises in the women's employment rate - account for changes in barriers to and costs associated with the changes of divorce rate over time.

Taken together, the social context and cultural understanding of reciprocity between partners influence and shape exchange behaviors in marriages, which have progressively changed over the past 30 years across cultures. As noted above, previous research findings indicate that intergenerational relationships play an important role in adult Korean children's marital quality, given the context of the patrilineal family system in Korea. On a macro level, Korean families are characterized as holding a collectivistic orientation and an emphasis on filial piety and support of the patrilineal family over the matrilineal family. The macro-level 
values result in husbands having more power and influence than wives and husbands' families being viewed as more influential than wives' families. Thus, it can be argued that cultural values and normative orientations are maintained across generations, and, at the same time, it is possible that new generations hold different values and normative orientations that characterize couples in a given society because of different practices and norms within social institutions over time. Therefore, items relating to intergenerational relationships in the revision of the MCLI could indicate different ecological realities for exchange relationships within the same culture as well as cultural value orientations between cultures. In doing so, the revised MCLI would provide support for whether the items comprising the measure are culturally universal or specific. Further, it could show distinctively different ecological realities for cohorts of individuals within exchange relationships in a given culture.

\section{The Ecological Context of Contemporary Families}

The following literature examines contemporary aspects of intimate relationships across cultures to focus on ecological factors that influence intimate exchanges. Given that the "grandparents" of marital assessments (e.g., the Dyadic Adjustment Scale, Spanier, 1976; the Locke-Wallace Marital Adjustment Test, Locke \& Wallace, 1959; the Marital Comparison Level index, Sabatelli, 1984) were first developed back in the 1950s and have been used with little modification since then, the development of modern marital assessment tools is needed. Thus, this section explores contemporary socio-cultural trends (i.e., technology use, work-life balance) that have occurred over the past decades.

Technology use. In recent years, there has been an explosion of technology use in everyday life. $77 \%$ of Americans had smartphones and nearly half of Americans owned a tablet computer in 2016 ("Record Share of Americans", 2017). In particular, Korea stands out as the country with the highest smartphone ownership rate, with $88 \%$ of respondents having 
one in 2017 (“10 Facts about Smartphones", 2017). Given the increased use of technology devices (e.g., smartphones, tablets, computers) in and around family life, many studies have investigated the impact of technology use on relational well-being (e.g., Chesley, 2005; McDaniel \& Coyne, 2016; Park, Lee, \& Chung, 2016; Roberts \& David, 2016). For example, Roberts and David (2016) developed a scale of partner phubbing (Pphubbing) that assesses the extent to which an individual is distracted by his/her cell phone while spending time with his/her partner. Phubbing is a word formed by combining "phone" and "snubbing." (Roberts \& David, 2016) and this scale was used to understand how Pphubbing impacts relationship satisfaction among romantic partners (Coyne, Stockdale, Busby, Iverson, \& Grant, 2011; Lenhart \& Duggan, 2014). Previous research suggests that interruptions and distractions caused by Pphubbing behaviors increase cell-phone related conflict and diminish partner's satisfaction with his or her relationship (Roberts \& David, 2016). Similarly, McDaniel and Coyne (2016) used the term "technoference," which refers to "everyday interruptions or intrusions in couple interactions due to technology devices" (McDaniel \& Coyne, 2016, p.85). This term can be used to describe any type of intrusions in couple interactions ranging from interruptions in face-to-face conversations to the feelings of intrusion when a partner checks a device even if their interactions were not happening at the exact moment. McDaniel and Coyne (2016) found that technoference is related to increased conflict over technology, which leads to feelings of relationship distress for women. Not surprisingly, most of the participants perceived that the use of a broad array of technologies (e.g., computers, smartphones, TV) interferes with their relationship with their partner. Moreover, in relation to work-to-family spillover, studies found that the use of cell phones blurs the boundaries between work and home, which leads to increased negative work-to-family spillover and lower satisfaction with family life (Chesley, 2005; Mazmanian, Orlikowski, \& Yates, 2005). Thus, it is possible that the use of technology devices interrupts couple interactions, which in 
turn, may lower intimacy or relationship satisfaction. Such components assessing the impact of technology use must be reflected in the revision of the MCLI due to their implications for contemporary marriages.

Time allocation of couples. Couples' time allocation seems critical to marital quality (Amato, Johnson, Booth, \& Rogers, 2007). For example, spending time together is positively related to happiness and meaning for both men and women, no matter what activities couples do together (Flood \& Genadek, 2016). The amount of time spent together seems also vital to marital quality, especially for those who have children (Claxton \& Perry-Jenkins, 2008; Roxburgh, 2006). Gager and Sanchez (2003) also found that wives' perception of time spent together is related to marital solidarity, buffering against divorce for women. However, given the context of long working hours in Korea (Hours worked, 2018) and the rise of dual-earner couples in both countries, the time couples spend together has come under duress in recent decades. Reductions in partner time may have negative impacts on relationship quality as the time couples spend together brings mutual feelings of closeness and relationship satisfaction. In considering time allocation as a constrained choice, both the amount of time spent together and how this time is spent need to be included in the revised MCLI as indicators of relationship quality to better understand the changing expectations and meaning of marital interaction in contemporary families.

\section{Spillover from work-related stress to relationship quality. In the past several} decades, a diverse scholarship on the intersection of work and family life has flourished with the rise in women's employment, the increase in work hours and job insecurity, and the introduction of new technologies. In particular, work and family conflict became a salient issue in family research, focusing on work-related stress and its effect on individuals and their families (Bianchi \& Milkie, 2010). Findings indicate that work-related stress does not 
directly impact relationship quality; however, it is associated with negative mood, which in turn, impacts the emotional climate of marital interactions (Lavee \& Ben-Ari, 2007; Roberts \& Levenson, 2001). Such negative spillover of stress from work to mood at home may reflect a deleterious effect of work-related stress on intimate relationships. Given that Korea is known for the longest work hours among developed countries (OECD, 2018), it is not surprising that workers are struggling with work-life balance and opting to pass up family time to spend more time in the office at night. As such, prevailing practices found in a given society influence how exchange relationships are organized and experienced. That is, the patterns of exchange found in family and intimate relationships within cultures are influenced by the practices present in the social institutions that contribute to the ecological niche of couples (Sabatelli, Lee, \& Ripoll-Núñez, 2018). In considering that the practices and norms within social institutions regulate individuals' access to the resources that they bring to their intimate relationships, the current ecological context, which emphasizes a strong commitment to employment, would play an important role in creating "exchange realities" in contemporary marriages.

Taken altogether, the changing practices found in the major institutions of society filter into how exchange relationships are experienced in a given time. With the rapid advancement of technology and increase in dual-earner couples, individuals from different cohorts may enter into exchange relationships with different resources and alternatives available to them. To capture sociocultural shifts in a given time and society, it is important to understand distinctly different ecological realities for cohorts of individuals within exchange relationships. These changes in ecological context imply that measurements should capture how exchange relationships are structured in the ever-changing world. Thus, I propose the contemporary aspects of marriages along with cultural-specific aspects of marital relationships (i.e., intergenerational exchanges) in the revisions of the MCLI. 


\section{Methodological Perspective on Cultural Revision}

\section{Cross-cultural Validation: Measurement Invariance Testing}

The MCLI was initially validated over the course of years by scholars from different cultural contexts, such as China, Hungary, and Korea (e.g., Attila, András, Tünde, \& Péter, 2014; Shek, Lam, Tsoi, \& Lam, 1993; Yang, 2004). The most common practice found in the cross-cultural validation of the MCLI was translation, back-translation, and evaluation of reliability and validity with their own samples. Further, in many instances, they have adapted the MCLI for use in their cultural contexts by adding culture-specific items to the original scale. For example, Yang (2004) added one item to the MCLI in her study of Korean couples: the amount of conflict with in-laws. Yang (2004) justified doing this because relationships with in-laws are thought to play a more influential role in how Korean couples experience their marriages when compared to contemporary U.S. couples. Similarly, the MCLI (Sabatelli, 1984) was adapted by Chan and Rudowicz (2002) for use with Chinese samples because the original scale did not reflect the indigenous characteristics of Chinese marriage. To capture marital relationships more adequately in a Chinese cultural context, they added 11 items to the original scale, including operational indicators of married respondents' relationships with parents, in-laws, friends, and children. However, using the MCLI in different cultural contexts in this way requires evidence that the measure captures marital quality in the same way across cultures. That is, we need evidence that the conceptualization and operationalization of a construct is invariant in spite of differences in cultural value orientations, customs, practices, and/or policies. This is the purpose of measurement invariance testing.

Despite the importance of measurement invariance that allows measures to be used for individuals from different cultures, the past literature using the MCLI in their studies 
included no measurement invariance testing procedure. This type of measurement study is particularly important to cross-cultural research, such as cross-cultural validation of a measure, which is the primary purpose of the present study. Determining whether the revised MCLI demonstrates measurement invariance would allow the revised MCLI to better serve its function of measuring marital quality across two cultures: Korea and the U.S. Thus, the present study tested measurement invariance, which is the degree to which the revised MCLI's measurement model functions in the same way across two cultures.

The most common statistical approach used to investigate measurement invariance is multiple group confirmatory factor analysis (MG-CFA) (Little, 1997; Vandenberg \& Lance, 2000). MG-CFA focuses on whether the assessment scale used is operating in the same way and the underlying construct has the same factorial structure and conceptual meaning across cultural groups (Byrne \& Campbell, 1999; Byrne \& van de Vijver, 2014; Kline, 2011). Exploratory factor analysis and confirmatory factor analysis (CFA) can be used in the initial formulation of a measurement model to define and refine the measurement model based on theory as well as empirical evidence (Dimitrov, 2010). Once the measurement model is defined, which includes a set of parameters that quantify the relationships among the measured and latent variables, a series of models for testing measurement invariance is followed (Meredith, 1993): configural, weak, strong, and strict invariance. Each model in the series requires that the factor structure, loadings, intercepts, and residuals respectively are constrained to be equal across groups.

Configural invariance. Configural invariance is a prerequisite step for testing a more restrictive measurement invariance model (i.e., weak and strong invariance). The parameters in this model can be estimated freely, which implies that no restrictions are placed. If configural invariance is supported, items of a scale have the same pattern of salient 
and nonsalient factor loadings across groups (Horn \& McArdle, 1992). On the other hand, evidence of noninvariance implies that there are different factor structures across groups (e.g., two factors emerge from one group, whereas a different number of factors characterizes another group). Configural invariance does not indicate that people across groups respond to the items in the same way; however, it reveals that respondents use the same conceptual framework to understand items.

Weak invariance. Weak invariance provides more restrictive testing by constraining the factor loadings to be equal across groups. The establishment of weak invariance implies that items load onto latent variables similarly across groups. In other words, the extent to which the latent variable predicts scores of indicator items is the same across groups (Vandenberg \& Lance, 2000). However, weak invariance does not indicate that the scales are invariant across groups because the testing only requires information about the covariation of the items; therefore, it is not tenable to compare means.

Strong invariance. Strong invariance allows for mean comparisons across groups. To examine strong invariance, equality constraints are placed on the intercepts, which tests whether the means of the observed items differ due to differences in the means of the underlying construct(s) across groups. If both the factor loadings and intercepts of the items are equal across groups, strong invariance is established (Meredith, 1993).

If weak invariance is established, then the invariance levels can be imposed on the factor variances and covariances. The invariance of the factor variances is supported when trait variances are equal across groups. The invariance of the factor covariances is established by testing whether latent covariances are equal across groups. If both the factor variances and covariances are satisfied, the correlations between the latent constructs are invariant across groups (Steenkamp \& Baumgartner, 1998). 
Strict invariance. Strict invariance testing requires equality amongst all the loadings, intercepts, and residual variances. This level of invariance implies that the effects of regression residuals for each indicator in the measurement model are the same across groups. Meredith (1993) argued that strict invariance should be achieved for a fair and equitable comparison. Additionally, $\mathrm{Wu}, \mathrm{Li}$, and Zumbo (2007) provided an exposition of the steps required to test strict invariance for detecting the existence of group-related systematic effect in residuals. However, this level of invariance is difficult to achieve empirically; therefore, most researchers recognize that weak or strong invariance would be sufficient evidence for measurement invariance (e.g., Little, 1997; Schmitt \& Kuljanin, 2008; Vandenberg \& Lance, 2000). In line with this, a review of measurement invariance testing literature found that very few studies conducted tests of residual equality in research practice (Schmitt \& Kuljanin, 2008; Vandenberg \& Lance, 2000). Thus, testing strict invariance seems to be a necessary step only if research questions are about an item's uniqueness (error variances and covariances). The invariance of item uniqueness across groups indicates that the items are measured with the same precision in each group.

Testing procedure. The invariance tests require a sequential process to examine the configural, weak, and strong invariance of a measure at the measurement and latent level. The tests compare between a hypothesized model in which parameters of interest are constrained to be equal across groups and a less restrictive model in which the same parameters are relaxed. In order to assess the effect of the equality constraint at each stage, the comparative fit index (CFI) and root mean square error of approximation (RMSEA) are inspected (Kline, 2011). In particular, Cheung and Rensvold (2002) suggested a criterion for evaluating a hypothesized invariance model after examining properties of 20 other goodness-of-fit indices proposed in the previous literature. They recommended that a change in the CFI of less than 0.01 would indicate that a hypothesized invariance holds. Given that this literature provides a 
basis for interpreting specific changes in CFI, this fit index was the primary criterion for determining measurement invariance in the present study. In addition, the RMSEA was used to assess model fit and changes in model fit in this study (Vandenberg \& Lance, 2000).

In measurement invariance analysis, configural invariance tests are conducted to estimate the pattern of free and fixed factor loadings as a first step. In other words, the same model is tested without imposing equality constraints for each group in this initial model. The importance of the configural invariance test is that it serves as the baseline against all subsequent tests exploring the question of measurement invariance. Multiple indices of model fit (e.g., CFI, TLI, RMSEA) are used to determine whether configural invariance holds (see Table 1). If configural invariance is supported, further restrictive constraints can be imposed for weak invariance. This second level of invariance, weak invariance, implies that the unit of measurement for the underlying factors (i.e., factor loading) is the same across groups. Lastly, the intercepts of the items are constrained to be equal across groups in order to establish strong invariance. If strong invariance holds across groups, comparison of latent factor means can be justified.

The measurement invariance testing presented above assumes that all individuals from the same culture are drawn from a single population, indicating there is a common pattern — a single set of "averaged" parameters — regardless of interindividual differences that exist within cultures. Such an approach has been tested in variable-centered analyses with an aim of assessing possible biases in measurements when applied across cultures. Evidence for measurement invariance is essential for meaningful cross-cultural comparisons; however, it only captures a snapshot of the "average relationship." In order to relax the assumption that all individuals are from the same underlying population and reflect subpopulations characterized by different set of parameters, person-centered analyses were also used in the 
present study.

Person-centered analyses identify particular constellations of characteristics-in the present study, marital quality — that describe subpopulations of married individuals within cultures presenting quantitatively distinct configurations on a set of indicators (i.e., item scores on the MCLI). Among the various person-centered analyses, Latent Profile Analysis (LPA) was used to detect similarities and differences across groups. Latent profiles are prototypical in nature, with each married individual having a probability of membership in a profile group based on their particular combinations of factors associated with general marital quality (Morin, Meyer, Creusier, \& Bietry, 2016). In particular, using both variable- and person-centered approaches is helpful for the investigation of psychometric properties because it provides a way to directly assess the extent to which a measurement model generalizes across unobserved subgroups of participants (Carter, Dalal, Lake, Lin, \& Zickar, 2011; Morin et al., 2016). In other words, measurement invariance testing in the variablecentered approach is useful in terms of detecting possible biases in instruments when applied to different cultural contexts. In contrast, an investigation of the similarities of latent profiles in the person-centered approach allows for the detection of similarities and differences across cultures, indicating the extent to which generalizability of profile solution deserves further exploration.

For this reason, the MG-CFA, which was the primary analytic approach used in this study, and LPA were used to investigate the extent to which research results generalizes across meaningful subgroups of married adults. Specifically, LPA was conducted after the appropriate level of measurement invariance had been established because a substantive interpretation of the profiles is meaningful only when the revised MCLI is reliably applicable to different cultural contexts. That is, such questions of population heterogeneity were of 
interest, but only after establishing the appropriate level of invariance.

\section{Conclusion}

The development of instruments that are culturally sensitive while being conceptually grounded and psychometrically sound is important for research on couples and families in countries outside the U.S., and in the instance of the present study, in Asian countries, to go forward. Thus, the primary goal of this study was to complete conceptual and psychometric revisions to the MCLI in a culturally appropriate way and test for evidence of the revised measure's cross-cultural relevance. The ecological/exchange framework that integrates cultural and historical factors into exchange relationships was both the impetus and foundation for this research. Guided by this framework, culture-specific aspects of marital relationships (i.e., intergenerational exchanges) were included and its content was reviewed by several expert Korean family researchers. Furthermore, based on the extensive literature regarding contemporary marriage and families, the revised MCLI took into consideration historical changes that have occurred over the past decades by including contemporary aspects of marriages in the revisions (e.g., technology use, changing time allocation, and work-to-relationship spillover). The current study, therefore, included culture-specific aspects as well as contemporary aspects of marriages in the revisions. In doing so, it explores and compares cultural similarities and differences in the measurement of marital relationships across two cultures: Korea and the U.S.

In sum, the adequacy of the conceptual definition of marital outcomes relative to expectations that was proposed by Sabatelli (1984) was not at issue here. The primary purpose was to improve the MCLI so that the existing scale measured the components of marital relations that are important to married individuals as defined by Sabatelli (1984) in a culturally relevant way for Korean married individuals. Furthermore, given the fact that the 
MCLI was developed more than three decades ago, it is important to update it in a more reliable and valid way for contemporary marriages. Therefore, the revisions also included the domains of contemporary marriages that may be missing in the original scale.

The second purpose of the present study was to examine whether certain factors (e.g., intergenerational relationships) developed indigenously in Korean cultures are relevant to Western cultures through a series of statistical analyses (i.e., measurement invariance testing and investigations of similarity of latent profiles). In doing so, results of the study would have the potential for explaining either systematic cultural differences or the cross-cultural relevance of the marital construct, which in turn, contributes to the advancement of marital research that provides better understandings of family dynamics in different sociocultural contexts. 


\section{CHAPTER THREE: METHODS}

The goal for this study is to develop a measure to assess marital quality that is culturally relevant and robust to be used to study Asian couples, with a particular emphasis on Korean couples. In order to accomplish this objective, the MCLI was refined to take a cross-cultural perspective into consideration. In other words, the present study was designed to explore both the continuum between the human universals and cultural specifics of marital constructs while, also, expanding our understanding beyond the existing constructs and measures that focus on Western families. The revision process began with restructuring the original scale in a multidimensional space to capture various dimensions of marital relationships. And then the culture-specific as well as contemporary aspects of marital relationships were included in the restructured scale. The revised MCLI was then examined in Korean and American samples by testing for measurement invariance. In doing so, this study would provide insight into how items/factors contained within the MCLI differ across cultures and a rationale for its use when applying the Western scale to different cultural contexts.

\section{Phase1: Revision of the MCLI}

The objective of the first phase was to identify the culture-specific as well as contemporary aspects of marital relationships and incorporate the existing factors that were previously included in the MCLI. To capture various dimensions of marital relationships, the underlying construct of the revised MCLI was defined within a multidimensional space. Although the original scale was intended to define marital complaints within a unidimensional space (Sabatelli, 1984), unidimensionality assumes that there is only one attribute of a construct, indicating every item in the measurement has the same attribute toward a common construct. It has some drawbacks, however, in that the salient dimension of 
marital experiences cannot be identified and measured. Also, the items on the original scale were unidimensional in content; in other words, each item only measures a single underlying construct.

Further, considering that everyone has a different expectation level based on personal experiences, beliefs, or cultural value orientations, it is important to assess how each individual perceives the salience of each dimension contained in the measure. Therefore, I treated marital quality as a general concept that encompasses specific aspects of individuals' perceptions of their marital relationship or particular kinds of marital complaints. This approach allows us to construct the various dimensions of marital relationships that are not combined into a single summary score. In other words, an individual's perception of the degree to which his or her marital expectations are being met includes a set of conceptually distinct but empirically correlated dimensions. For example, even if individuals showed the same average score of marital quality, they can exhibit a variety of profiles, such as being low on conflict management and high on sexual intimacy or alternatively, high on conflict management and low on sexual intimacy. Assessing multiple dimensions of marital quality, which are conceptually distinct but empirically correlated, allows us to disentangle what areas of marital relationships are more salient than others. Therefore, phase 1 included the reorganization of the original scale in a way that taps into several specific marital behaviors and evaluations (e.g., emotional and sexual intimacy), in addition to generating items that are salient for Korean married couples and relevant to contemporary marriages across cultures.

First, the MCLI was restructured within the multidimensional space based on the content of the individual items. The measure included 32 items that each tapped one of five distinct domains: (a) emotional intimacy, (b) sexual intimacy, (c) marital conflicts, (d) agreement about different areas of relationships, and (e) instrumental aspects of relationships. 
The response format was a 7-point Likert scale, with the midpoint reflecting the individual's expectation level. Scores ranged from 1 (much worse than you expect) to 7 (much better than you expect).

Emotional intimacy consisted of thirteen items. The items asked participants to what extent their emotional needs are met. Examples of the items were as follows: "the amount of love you get from your spouse," "the amount of commitment you experience from your spouse," and "the amount of your spouse is trusting of you." For all items, higher scores reflected a more favorable evaluation of outcomes relative to expectations.

Sexual intimacy consisted of four items. The items pertained to the extent participants' sexual needs are met. Examples of the items were as follows: "the degree of physical attractiveness of your spouse," "the amount of interest in sex your spouse expresses," and "the amount of sexual activity that you experience." For all items, higher scores indicated that sexual intimacy exceeds participants' expectations.

Marital conflicts consisted of six items. The items pertained to the amount of conflict in the relationship. Examples of the items were as follows: "the amount of conflict over daily decisions that exists," "the amount of arguing over petty issues that you experience," and "the amount of conflict over the use of leisure time that you experience." For all items, higher scores reflected higher levels of conflicts.

Agreement about different areas of relationships consisted of four items. The items asked participants to rate the extent of their agreement about different areas of the relationship. Examples of the items were as follows: "the amount of disagreement over friends you experience" and "the degree to which you and your spouse agree on the number of children to have.” For all items, higher scores reflected higher levels of agreement. 
Instrumental aspects of relationships consisted of five items. The items asked participants to rate instrumental support from their partners. Examples of the items were as follows: "the degree to which your financial needs are met by your relationship," "the amount to which your spouse supports your choice of an occupation," and "the amount of responsibility your spouse accepts for household tasks."

The next step in the revisions of the MCLI was to operationalize constructs that tapped into cultural and contemporary marital issues that were not included in the original version of the measure. Based on the emerged themes from a comprehensive literature review, two additional constructs were integrated into the measure: intergenerational relationships and perceived problems in contemporary marriages. I initially developed 18 items for the two constructs of the revised MCLI. After the generation of the items, I had several discussions with the author of the original scale to ensure the overall operationalization of the constructs as well as the content of the individual items. Through the discussions, item wordings were revised and two items were omitted. Finally, 16 items were included in the content validation for the next step. The intergenerational relationships construct reflected the degree to which individuals have intergenerational exchanges (e.g., "the amount of contact that you have with in-laws," "the amount of conflict you experience with your spouse over issues involving your in-laws", and "the amount of conflict you experience with your spouse over issues involving your parents"). The construct of perceived problems in contemporary marriages assessed complaints of partners' lifestyle (e.g., "the degree to which your spouse's work-related stress affects your relationship," "the amount of time your spouse spends on electronic devices," and "the amount of time your spouse spends on electronic devices when you both are supposed to being doing things together"). Thus, I used the label complaints about partner's lifestyle to describe this construct. 
Content validation was completed by two groups of reviewers. First, content experts were selected based on their expertise and professional experiences in Family Studies in Korea. In addition to the original 32 items, 16 new items were submitted to three experts on Korean families, each having more than 10 years of professional experience in Korean Family Studies. The experts were instructed to rate each item on the extent to which it was relevant, contained social desirability, or overlapped substantially with other items listed, and to provide written feedback if needed. Based on their responses, 14 new items were selected for inclusion and combined with the 32 original items to form a revised MCLI. The revised MCLI was then administered to ten Korean married adults and five Americans to assess the relevance of the 46 items to the given constructs.

For the Korean version of the MCLI, I started from a validated English version and translated it into the Korean version using a formal translation back-translation method conducted by independent bilingual experts (Brislin, 1986). First, all items were translated into Korean by the author and then into English again by three bilingual psychologists to check the appropriateness of the translation. During the translation process, the Korean measure was further administered to five Korean married adults who were from diverse backgrounds (e.g., education level, age) to detect and modify the items that were less clear and not easily understood. Based on the content validation from experts and married individuals in both countries, the 46-item version of the MCLI was created for phase 2, crosscultural validation of the revised scale.

\section{Phase2: Cross-cultural Validation of Measure}

Data collection procedure. After IRB approval, I recruited married individuals in 
Korea and U.S. to collect data for measurement invariance testing through Embrain ${ }^{1}$ and Amazon Mechanical Turk ${ }^{2}$. Embrain provided data collection services with an online panel in Korea. Similarly, the Mechanical Turk provided an online survey platform and screened a participant pool according to the inclusion criteria in the U.S. Small financial incentives ${ }^{3}$ were offered to the respondents in return for the completion of a survey in both countries. The survey included the revised MCLI, fifteen demographic questions, relationship satisfaction, and relationship instability. All questionnaires were translated into Korean for Korean participants.

Study participants. Participants in this study were married adults in Korea and the U.S. A total of 322 Korean and 354 American participants were included in the analyses.

Table 1 reports the demographic characteristics of the study sample.

Korean participants. The Korean sample consisted of 322 married adults. Ages of participants ranged from 25 to 59 years, with a mean age of $41.15(S D=7.57)$. Most of the participants were college graduates $(71.4 \%)$ and were employed (78.9\%). Average household income was in the range of $\$ 60,000$ to $\$ 70,000 /$ year. The average length of marriage was 11.9 years $(S D=8.84$, range $=1-35)$. Most of them were parents $(81 \%)$ raising more than one child.

American participants. The U.S. sample consisted of 354 married adults. Gender composition showed that the disparity between females and males was larger in the U.S.

\footnotetext{
1 Embrain is an online research agency with the largest consumer panel in Korea.

2 The Mechanical Turk is a participant recruitment tool created by Amazon, composed of more than 500,000 individuals from 190 countries (Paolacci \& Chandler, 2014). Payment, which would be cents rather than dollars, allows them to hire "workers" for the completion of surveys on the Internet. In particular, MTurk has been used to conduct cross-cultural studies of decision making (e.g., Eriksson \& Simpson, 2010). Moreover, empirical studies have shown that internet samples are more diverse than those from traditional methods in terms of gender, socioeconomic status, geographic region, and age (Buhrmester, Kwang, \& Gosling, 2011; Gosling, Vazire, Srivastava, \& John, 2004).

3 The author paid for the company's service at the rate of 2 U.S. dollars per one participant in both countries. The service cost included incentives for the participants. The company, not the author, compensated participants who completed the survey, according to the company's internal policy.
} 
sample, with $62.4 \%$ female, than the Korean sample, which was evenly split. Ages of the participants ranged from 20 to 73 years, with a mean age of $38.32(S D=10.24)$. The participants in the sample were predominantly White, with less than $15 \%$ Hispanic, Black, or Asian. The educational level of participants fell into two groups: those that were high school graduates or had partial college/specialized training and those that were college graduates or had received graduate training. Average household income was in the range of $\$ 58,000$ to $\$ 65,000 /$ year. The average length of marriage was 12.25 years $(S D=9.49$, range $=1-49)$. Sixty-seven percent of the participants were parents raising more than two children.

Table 1. Participants Demographic Characteristics

\begin{tabular}{|c|c|c|}
\hline Variables & $\begin{array}{c}\text { Koreans }(n=322) \\
N(\%)\end{array}$ & $\begin{array}{c}\text { Americans }(\mathrm{n}=354) \\
\mathrm{N}(\%)\end{array}$ \\
\hline \multicolumn{3}{|l|}{ Gender } \\
\hline Male & $161(50 \%)$ & $133(37.6 \%)$ \\
\hline Female & $161(50 \%)$ & $221(62.4 \%)$ \\
\hline \multicolumn{3}{|l|}{ Being a parent } \\
\hline Yes & $261(81.1 \%)$ & $238(67.2 \%)$ \\
\hline No & $61(18.9 \%)$ & $116(32.8 \%)$ \\
\hline \multicolumn{3}{|l|}{ Education } \\
\hline Some high school & $2(0.6 \%)$ & $4(1.1 \%)$ \\
\hline Completed high school & $30(9.3 \%)$ & $36(10.2 \%)$ \\
\hline Some college & $60(18.7 \%)$ & $128(36.1 \%)$ \\
\hline Completed college & $192(59.6 \%)$ & $138(39 \%)$ \\
\hline Completed graduate degree & $38(11.8 \%)$ & $48(13.6 \%)$ \\
\hline \multicolumn{3}{|l|}{ Employment status } \\
\hline Employed full-time & $223(69.3 \%)$ & $222(62.7 \%)$ \\
\hline Employed part-time & $31(9.6 \%)$ & $44(12.4 \%)$ \\
\hline Unemployed & $57(17.7 \%)$ & $56(15.8 \%)$ \\
\hline Retired & $4(1.2 \%)$ & $12(3.4 \%)$ \\
\hline Other (e.g., student) & $7(2.2 \%)$ & $20(5.6 \%)$ \\
\hline \multicolumn{3}{|l|}{ Income } \\
\hline Less than $\$ 20,000$ & $24(7.5 \%)$ & $117(33.1 \%)$ \\
\hline$\$ 20,000-\$ 35,000$ & $60(18.6 \%)$ & $66(18.6 \%)$ \\
\hline$\$ 35,000-\$ 50,000$ & $94(29.2 \%)$ & $53(15 \%)$ \\
\hline$\$ 50,000-\$ 75,000$ & $101(31.4 \%)$ & $78(22 \%)$ \\
\hline$\$ 75,000-\$ 100,000$ & $34(10.6 \%)$ & $22(6.2 \%)$ \\
\hline More than $\$ 100,000$ & $9(2.8 \%)$ & $18(5.1 \%)$ \\
\hline \multicolumn{3}{|l|}{ Ethnicity } \\
\hline White & N/A & $293(82.8 \%)$ \\
\hline Black & & $8(2.3 \%)$ \\
\hline Hispanic & & $21(5.9 \%)$ \\
\hline
\end{tabular}




\begin{tabular}{lcc}
\hline Asian & & $23(6.5 \%)$ \\
Other & & $9(2.6 \%)$ \\
\hline Variables & $M(S D)$ & $M(S D)$ \\
\hline Age & $41.15(7.57)$ & $38.32(10.24)$ \\
Relationship duration (years) & $11.90(8.84)$ & $12.25(9.49)$ \\
Number of children living together & $1.76(.91)$ & $2.03(.98)$ \\
\hline
\end{tabular}

Measures. The measures used in this study included demographic questions, relationship satisfaction, and relationship stability, and the revised 46-item MCLI (See Appendix).

Demographic questionnaire. General demographic information was collected including age, education, employment status, participant's and partner's income, number of children, and duration of marriage.

Relationship satisfaction. Four items asked respondents to state whether they were satisfied with their marriage, partner, relationship with partner, and sex life. The items rated on a 5-point Likert scale ranging from being extremely dissatisfied to extremely satisfied. Cronbach's alpha was .93 for Koreans and .90 for Americans.

Relationship stability. The five-item relationship stability scale was used to assess whether thoughts and behaviors associated with relationship dissolution had occurred recently (Booth, Johnson, \& Edwards, 1983). The items were rated on a dichotomous scale: yes and no. The Kuder-Richardson 20 (KR20) was .85 for Koreans and .79 for Americans.

Revised MCLI. The 46-item MCLI was used to assess how their relationship outcomes from their marriage compare with their unique expectations. Item responses range from "much worse than I expect" (-3) to "much better than I expect" (+3). A scale score of -3 was converted to 1 and +3 was assigned to 7 in the following analysis. Higher scores on this scale indicate higher levels of marital satisfaction and lower levels of complaints. Cronbach's 
alpha was .97 for Koreans and .96 for Americans. Table 2 shows the items of the revised MCLI.

Table 2. Factors and Items on the revised MCLI

\begin{tabular}{|c|c|c|}
\hline Factor & $\begin{array}{l}\text { \# of } \\
\text { items }\end{array}$ & Item \\
\hline $\begin{array}{l}\text { Emotional } \\
\text { intimacy }\end{array}$ & 13 & $\begin{array}{l}\text { Item 1: The amount of love you get from your spouse } \\
\text { Item 2: The amount of compatibility that you experience } \\
\text { Item 3: The amount of mutual respect you experience } \\
\text { Item 4: The degree to which your emotional needs are met } \\
\text { by your spouse } \\
\text { Item 7: The amount of affection your spouse displays } \\
\text { Item 8: The amount of commitment you experience from } \\
\text { your spouse } \\
\text { Item 9: The amount your spouse is willing to listen to you } \\
\text { Item 10: The degree to which your interpersonal } \\
\text { communications are effective } \\
\text { Item 11: The amount of companionship you experience } \\
\text { Item 12: The amount of relationship equality you experience } \\
\text { Item 13: The amount of confiding that occurs between you } \\
\text { and your spouse } \\
\text { Item 14: The amount your spouse is trusting of you } \\
\text { Item 16: The amount of time you spend together }\end{array}$ \\
\hline Sexual intimacy & 5 & $\begin{array}{l}\text { Item 5: The degree to which your sexual needs are met by } \\
\text { your spouse } \\
\text { Item 17: The degree of physical attractiveness of your } \\
\text { spouse } \\
\text { Item 19: The amount of interest in sex your spouse expresses } \\
\text { Item 21: The amount of sexual activity that you experience } \\
\text { Item 24: The amount that you and your spouse discuss sex }\end{array}$ \\
\hline Marital conflicts & 6 & $\begin{array}{l}\text { Item 18: The amount of conflict over daily decisions that } \\
\text { exist } \\
\text { Item 20: The amount of arguing over petty issues that you } \\
\text { experience } \\
\text { Item 22: The amount of conflict over the use of leisure time } \\
\text { that you experience } \\
\text { Item 23: The amount of criticism your spouse expresses } \\
\text { Item 30: The amount of conflict over money you experience } \\
\text { Item 31: The amount of jealousy your spouse expresses }\end{array}$ \\
\hline $\begin{array}{l}\text { Agreement } \\
\text { about different } \\
\text { areas of } \\
\text { relationships }\end{array}$ & 5 & $\begin{array}{l}\text { Item 26: The amount of disagreement over friends you } \\
\text { experience } \\
\text { Item 27: The amount of freedom you experience in pursuing } \\
\text { other friendships } \\
\text { Item 32: The amount of privacy you experience } \\
\text { Item 33: The degree to which you and your spouse agree on } \\
\text { the number of children to have }\end{array}$ \\
\hline
\end{tabular}




\begin{tabular}{|c|c|c|}
\hline & & $\begin{array}{l}\text { Item 35: The amount of conflict over issues involving } \\
\text { religious activity }\end{array}$ \\
\hline $\begin{array}{l}\text { Instrumental } \\
\text { aspects of } \\
\text { relationships }\end{array}$ & 6 & $\begin{array}{l}\text { Item 6: The degree to which your financial needs are met by } \\
\text { your relationship } \\
\text { Item 15: The fairness with which money is spent } \\
\text { Item 25: The amount to which you and your spouse agree on } \\
\text { your lifestyle } \\
\text { Item 28: The amount to which your spouse supports your } \\
\text { choice of an occupation } \\
\text { Item 29: The degree to which the responsibility for } \\
\text { household tasks is shared } \\
\text { Item 34: The amount of responsibility your spouse accepts } \\
\text { for household tasks }\end{array}$ \\
\hline $\begin{array}{l}\text { Intergenerational } \\
\text { relationships }\end{array}$ & 7 & $\begin{array}{l}\text { Item 36: The amount of contact that you have with in-laws } \\
\text { Item 37: The amount of conflict you experience with your } \\
\text { spouse over issues involving your in-laws } \\
\text { Item 38: The amount of conflict you experience with your } \\
\text { spouse over issues involving your parents. } \\
\text { Item 39: The degree of financial support you receive from } \\
\text { your parents } \\
\text { Item 40: The degree of financial support you receive from } \\
\text { your in-laws } \\
\text { Item 41: The degree of financial support you provide to your } \\
\text { parents } \\
\text { Item 42: The degree of financial support you provide to your } \\
\text { in-laws }\end{array}$ \\
\hline $\begin{array}{l}\text { Complaints } \\
\text { about partner's } \\
\text { lifestyle }\end{array}$ & 4 & $\begin{array}{l}\text { Item 43: The degree to which your spouse's work-related } \\
\text { stress affects your relationship } \\
\text { Item 44: The amount of time your spouse spends on } \\
\text { electronic devices (e.g., smartphone, tablet PC, } \\
\text { laptop/computer, TV, Play Station, etc.) } \\
\text { Item 45: The amount of time your spouse spends on } \\
\text { electronic devices (e.g., smart phone, tablet PC, } \\
\text { laptop/computer, TV, Play Station, etc.) when you both are } \\
\text { supposed to being doing things together } \\
\text { Item 46: The amount of time your spouse spends working }\end{array}$ \\
\hline
\end{tabular}

Data analysis. As noted in the literature review above, there are two approaches to the systematic quantitative comparisons of a measure used across cultures: variable- and person-centered approaches. The variable-centered approach has been used widely in crosscultural studies, such as multi-group confirmatory factor analysis (i.e., measurement invariance testing). Such an analysis is the primary analytic approach in the present study; 
however, it operates under the assumption that all individuals are from a single population. To relax this assumption, the present study also used the person-centered approach, which assumes that the sample reflects multiple subpopulations characterized by a different set of parameters. Furthermore, given that the primary goal of the study was to investigate psychometric properties of the measure for meaningful cross-cultural comparison, I assessed the extent to which relatively homogenous subpopulations (i.e., latent profile) generalize across cultures. That is, LPA in multi-group analysis allows tests of the similarity of profile solutions (e.g., similarity of the indicators' means and variances across groups) across subpopulations. In doing so, we can gain insights into the diverse and unique qualities of different marital relationships within and between cultures. Such studies guide meaningful comparisons of profile similarity across cultures, which in turn, provide the extent to which a measurement model generalizes across unobserved subgroups of married adults from Korea and the U.S.

Considering that the goal of the present study was to validate the revised MCLI across cultures, the primary analytic approach was multi-group confirmatory factor analysis (i.e., measurement invariance testing) in the variable-centered approach. After establishing the appropriate level of invariance, the person-centered analysis (i.e., LPA) was conducted to detect relatively homogenous subpopulations of married adults that show qualitatively and quantitatively distinct configurations on a set of MCLI items. Based on a profile solution in each group, profile similarity across groups was investigated. In sum, the similarity of profiles across cultures was of interest, assessing the extent to which a latent profile solution replicates across cultures.

Analytic procedure for measurement invariance testing. First, confirmatory factor analysis (CFA) was used to examine the latent structure of the measure in both samples. The 
goal of CFA was to evaluate how well each specified dimension adequately described the data. In CFA, the researcher can specify a priori, that is, the number of factors is prespecified based on theory or prior research evidence. Furthermore, CFA allows empirical comparison of the goodness of fit across several alternative models to revise and improve the model based on theoretical and empirical evidence to arrive at an adequately fitting model (Brown, 2006). Given that the focus of this study was to test the degree to which the revised MCLI's measurement model functions in the same way across cultures, a particular measurement model that is employed must be the same for each group. Therefore, the hypothesized measurement model with seven factors fit the data in both samples. The purpose of the CFA analyses was to assess the fit of the starting measurement model in both groups. In order to assess model fit between the initial and respecified models, multiple indices were used to evaluate model fit, such as CFI, TLI, and RMSEA (Brown, 2006). See Table 3 for optimal values for these indices.

Table 3. A summary of Criteria for Evaluating Model Fit

\begin{tabular}{ll}
\hline Fit Index & Criteria for model fit \\
\hline Root mean square error of approximation & $<.05$ for good fit \\
(RMSEA) & $<.08$ for acceptable fit \\
Comparative fit index (CFI) & $>.95$ for good fit \\
Tucker-Lewis index (TLI) & $>.95$ for good fit \\
Standardized root mean square residual (SRMR) & $<.08$ for good fit \\
\hline
\end{tabular}

In order to estimate the parameters in CFA, the measurement model must be identified based on known information (i.e., the variances and covariances in the sample) to obtain a unique set of parameter estimates for unknown values (e.g., factor loadings, factor correlations) in the model (Brown, 2006). According to Brown (2006), model identification is reached when a model with one factor has at least three indicators. However, the hypothesized measurement model included seven constructs for forty six items; in particular, 
the emotional intimacy factor included thirteen items from the original scale. Considering that several items composing the emotional intimacy factor dealt with the same issues, such as affection and companionship between partners, I created parcels that are "aggregated-level [indicators] comprised of the sum (or average) of two or more items, responses, or behaviors" (Little, Cunningham, Shahar, \& Widaman, 2002, p.152). Using parcels has several advantages over items in the context of invariance testing because "the unique variances of items are minimized and the ratio of true-score variance to error variances is improved" (Little, Rhemtulla, Gibson, \& Schoemann, 2013, p.294). Furthermore, given that the goal of the present study was to understand the latent construct of marital quality and its relation to other constructs rather than understanding the structure of the items, parceling was beneficial for making the measurement model a parsimonious representation of the construct. Thus, I combined these items into parcels containing two or more items and used the resulting aggregate scores as manifest indicators of a latent construct. Specifically, drawing from Little et al.'s (2013) suggestions, I assigned items with a correlated residual to the same parcel based on an item-level solution, which was the most advantageous parcel solution. This parceling strategy resulted in ten item parcels being included in the following analyses (See Table 4 for items used to create parcels).

Table 4. Item Parcels

\begin{tabular}{|c|c|c|}
\hline Assigned factor & Parcel \# & Item \# \\
\hline \multirow[t]{3}{*}{$\begin{array}{l}\text { Emotional } \\
\text { intimacy }\end{array}$} & $\mathrm{P} 1$ & $\begin{array}{l}\text { Item 1: The amount of love you get from your spouse } \\
\text { Item 4: The degree to which your emotional needs are met } \\
\text { by your spouse } \\
\text { Item 7: The amount of affection your spouse displays } \\
\text { Item11:The degree to which your interpersonal } \\
\text { communications are effective }\end{array}$ \\
\hline & $\mathrm{P} 2$ & $\begin{array}{l}\text { Item 3: The amount of mutual respect you experience } \\
\text { Item 13: The amount of confiding that occurs between you } \\
\text { and your spouse }\end{array}$ \\
\hline & P3 & $\begin{array}{l}\text { Item 8: The amount of commitment you experience from } \\
\text { your spouse } \\
\text { Item 14: The amount your spouse is trusting of you }\end{array}$ \\
\hline
\end{tabular}




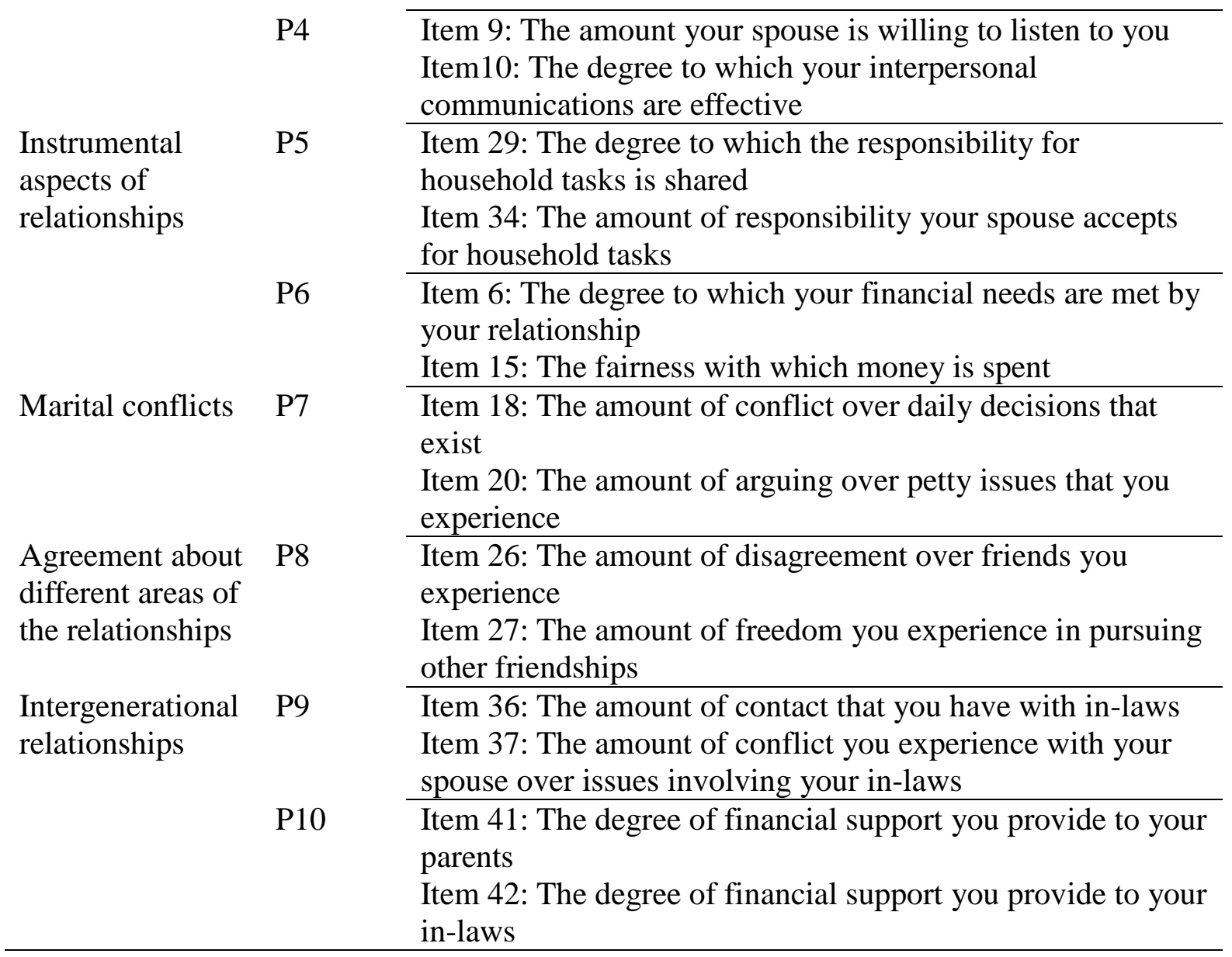

Furthermore, I hypothesized a higher-order factor model that imposes a more parsimonious structure to account for the interrelationships among factors established by CFA (Brown, 2006). The second-order model allows for the examination of the hypothesis that several constructs that are related, but distinct from each other, can be accounted for by underlying higher-order constructs. This model can apply when "(a) the lower order factors are substantially correlated with each other and (b) there is a higher order factor that is hypothesized to account for the relations among the lower order factors (Chen, Sousa, \& West, 2005, p.473)." Statistical tests of the fit of a hypothesized second-order factor normally require at least three lower-order factors (Kline, 2011). The advantage of using a secondorder factor model is that the model can test whether the hypothesized higher order factor actually accounts for the pattern of relations between the lower order factors. Therefore, the 
second-order factor model was utilized to identify whether the specific factors can predict external criteria over and above the general factor in the following measurement invariance testing.

Applying the rationale for the exploration of a second-order factor model as outlined by Chen and colleagues (2005) the hypothesized model in the present study is presented in Figure 1. To test whether there is a general marital quality factor that underlies specific relational dimensions derived from marital complaints, I hypothesized that seven relational dimensions, which were each assessed by multiple items, were lower-order factors, and the general marital quality was a higher-order factor, which accounted for the commonality among the specific relational dimensions. In doing so, the contribution of each dimension to a higher-order factor was assessed and delineated, which in turn, ascertained the salient dimension of marital experiences across cultures. 
Figure 1. Hypothesized model

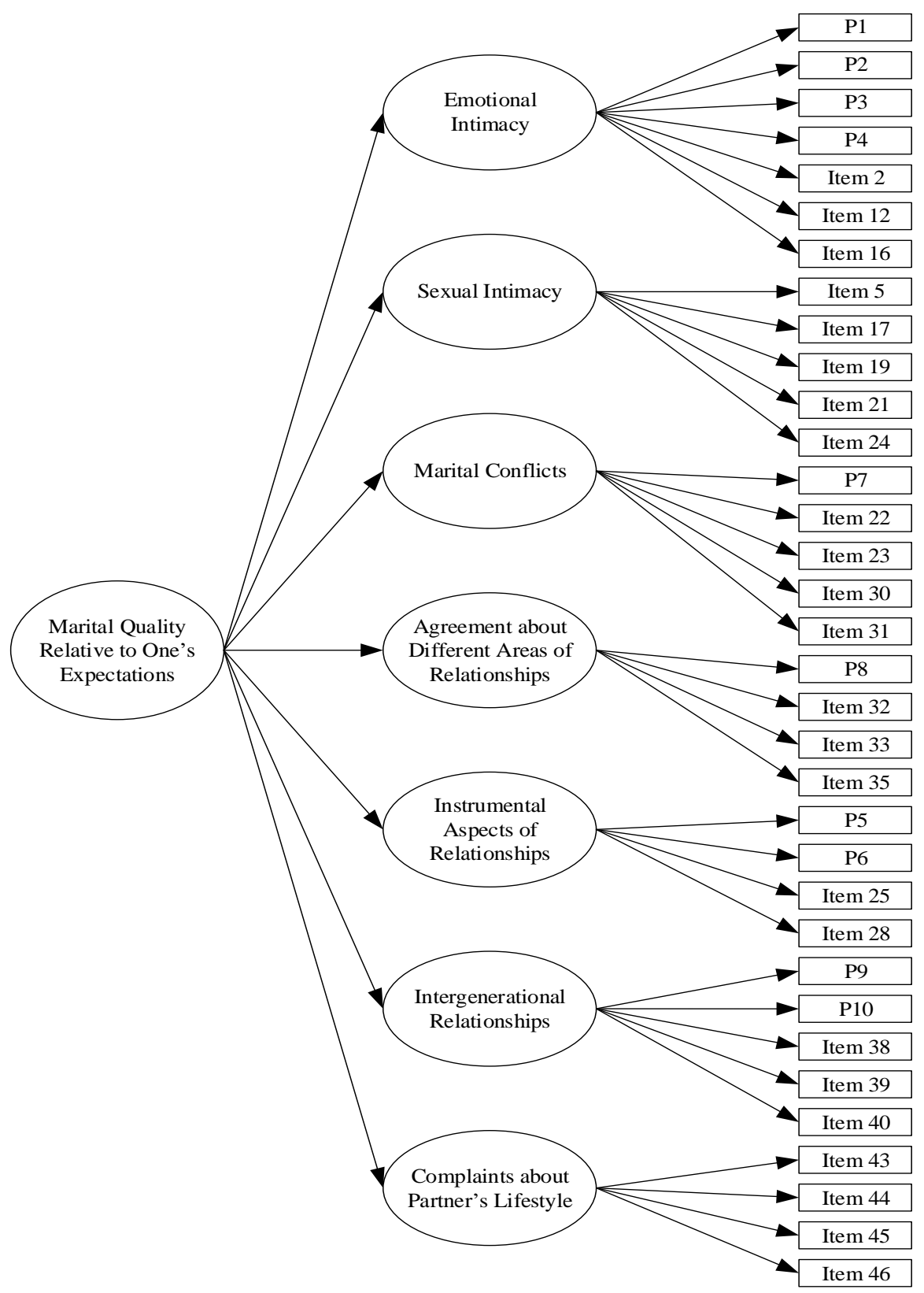

Because of the second-order factor structure of the hypothesized model, I extended the invariance testing procedures according to the recommendations of Chen and colleagues (2005). Specifically, weak, strong, and strict invariance need to be tested on the first-order factor level, as well as on the second-order factor level, which results in testing seven instead of four models (See Table 5 for a summary of the steps). As noted above, the two major criteria for assessing changes in model fit were the changes in CFI and RMSEA (Cheung \& 
Rensvold, 2002).

Table 5. Levels of Measurement Invariance for Second-order Factor Model

\begin{tabular}{|c|c|c|}
\hline $\begin{array}{l}\text { Level of } \\
\text { Invariance }\end{array}$ & \multicolumn{2}{|c|}{ Description } \\
\hline Configural & \multicolumn{2}{|c|}{ Model 1: Same factor structure across groups } \\
\hline & $\begin{array}{l}\text { First-order factor: } \\
\text { Specific dimensions }\end{array}$ & $\begin{array}{l}\text { Second-order factor: } \\
\text { marital quality }\end{array}$ \\
\hline Weak invariance & $\begin{array}{l}\text { Model 2: Invariance of first- } \\
\text { order factor loadings }\end{array}$ & $\begin{array}{l}\text { Model 3: Invariance of second- } \\
\text { order factor loadings }\end{array}$ \\
\hline Strong invariance & $\begin{array}{l}\text { Model 4: Invariance of } \\
\text { intercepts of items }\end{array}$ & $\begin{array}{l}\text { Model 5: Invariance of intercepts } \\
\text { of first-order factors }\end{array}$ \\
\hline Strict invariance & $\begin{array}{l}\text { Model 7: Invariance of residual } \\
\text { variances of items }\end{array}$ & $\begin{array}{l}\text { Model 6: Invariance of } \\
\text { disturbances of first-order factors }\end{array}$ \\
\hline
\end{tabular}

\section{Additional procedure for multi-group analysis of similarity in latent profile}

solutions. Latent profile analyses were conducted based on the second-order factors' scores reflecting specific domains of marital quality (e.g., emotional intimacy, intergenerational relationships) using the robust maximum likelihood (MLR) estimator available in Mplus 8 (Muthén \& Muthén, 1998-2017). Rather than using latent variables (i.e., the items/item parcels used to estimate latent factors) to estimate the relationship profiles, the means of second-order factors were used in LPA. Given the complexity of mixture models including LPAs, factor scores obtained from preliminary measurement models have been frequently used in practice (e.g., Kam et al., 2016; Morin et al., 2016), therefore, I used the means of second-order factors as the profile indicators.

The best fitting model was chosen using the Akaike Information Criteria (AIC), the Bayesian Information Criteria (BIC), the sample-adjusted BIC (SABIC), the Lo, Mendell, and Rubin (2001) likelihood ratio test (LMR), and the Bootstrap Likelihood Ration Test (BLRT). Overall, smaller values on the AIC, BIC, and SABIC indicate a better fit and both 
the LMR and BLRT are used to compare a $k$ profile model with a $k-1$ profile model. A significant $p$ value indicates a $k$ profile model should be retained over a $k-1$ profile model. Simulation studies have shown that the BIC, SABIC, and BLRT are more effective in choosing the best fitting model than the AIC (e.g., Nylund, Asparouhov, \& Muthén, 2007; Tofighi, \& Enders, 2008). Therefore, the AIC was only reported to ensure a thorough class enumeration. Also, the entropy that shows greater confidence in appropriate classification was examined. The entropy ranges 0 to 1 with higher values indicating classification accuracy. The number of profiles was determined by fitting models sequentially.

The first step in the person-centered approach examines whether the same number of profiles can be identified across cultures, which is configural similarity. The class enumeration procedure is conducted separately across groups. It serves as a baseline comparison model for the subsequent steps of similarity for multiple group LPA. When established, a multiple group LPA model can be estimated to test whether the indicators' means, variances, and relative sizes of the profiles are the same across groups (See Table 6 for a summary of the steps). Furthermore, the relations between covariates (i.e., predictors, outcomes) and profiles can be estimated to establish the construct validity of extracted profiles (Morin et al., 2016; Muthén, 2003).

Table 6. Sequences of Tests of Similarity for Multi-Group LPA

\begin{tabular}{|c|c|c|}
\hline $\begin{array}{l}\text { Profile } \\
\text { similarity }\end{array}$ & Description & Prerequisite \\
\hline $\begin{array}{l}\text { Model 1: } \\
\text { Configural }\end{array}$ & Same number of latent profiles across groups & None \\
\hline $\begin{array}{l}\text { Model 2: } \\
\text { Structural }\end{array}$ & Equality of within profile means across groups & Model 1 \\
\hline $\begin{array}{l}\text { Model 3: } \\
\text { Dispersion }\end{array}$ & Equality of within profile variances across groups & Model $1 \& 2$ \\
\hline $\begin{array}{l}\text { Model 4: } \\
\text { Distributional }\end{array}$ & $\begin{array}{l}\text { Similarity of sizes of all profiles (i.e., class probabilities) } \\
\text { across groups }\end{array}$ & Model 1 \\
\hline $\begin{array}{l}\text { Model 5: } \\
\text { Predictive }\end{array}$ & $\begin{array}{l}\text { Equality of the effects of predictors across groups; } \\
\text { predictors added to the most similar model from previous } \\
\text { steps while ensuring that they do not change the nature of }\end{array}$ & Model $1 \& 2$ \\
\hline
\end{tabular}




\begin{tabular}{lll}
\hline Model 6: & the profiles & Equality of the levels outcomes across groups; outcomes \\
Explanatory & $\begin{array}{l}\text { added to the most similar model from previous steps while } \\
\text { ensuring that they do not change the nature of the profiles }\end{array}$ & Model \& 2 \\
&
\end{tabular}

Descriptive statistics were analyzed using SPSS Version 22 (IBM Corp.).

Measurement invariance analyses and latent profile analyses were conducted in Mplus 8 (Muthén \& Muthén, 1998-2017). 


\section{CHAPTER FOUR: RESULTS}

This chapter presents results of the study. The first section presents the results of the phase 1 study to address the first research question: What are the dimensions of the revised MCLI? The purpose of this portion of the study was to estimate the basic measurement model in each group to determine whether the model fits well for Koreans and Americans separately, which was also the first step in measurement invariance testing. Next, the results of a series of MG-CFAs are presented to address the second research question: To what extent can the dimensions of the MCLI be used across cultures? As described in Chapter Three, measurement invariance testing followed a sequenced testing procedure guided by Chen and colleagues (2005). Tests of measurement invariance in a second-order factor model considered equality of first-and second-order factor loadings, intercepts of measured variables, intercepts of first-order factors, disturbances of first-order factors, and residual variances of observed variables. Also, I present the results of the additional analyses to further examine the similarity of profile solutions across groups using person-centered analyses.

\section{First-Order CFA Model}

The hypothesized model included seven factors in first-order models; no items crossloaded and there were no correlated errors. For the purpose of model identification, the factors were scaled by fixing the variance of each factor to one. The hypothesized model was estimated to determine whether the model fit well for Koreans and Americans separately. The results of this estimation indicated a poor fit (see Table 7). All fit indices in each group fell below the range associated with a good fit. Furthermore, the correlations between the Agreement about different areas of relationships and Instrumental aspects of relationships factors were above .85 in both groups, which is often used as a cutoff criterion for poor 
discriminant validity (Brown, 2006). In addition to this, the correlations between the Emotional intimacy and Instrumental aspects of relationships factors were high in both groups, .96 and .93 for Koreans and Americans, respectively. This is likely to happen when factors overlap; in other words, these factors do not represent different and distinct constructs. This is a threat to parsimony and interpretability of the CFA model. In order to improve parsimony, combining the highly overlapping factors or dropping one of the factors that is defined by only a few indicators or has limited variance is recommended (Brown, 2006).

In addition to the re-specification regarding the number of factors, empirical evidence (i.e., modification indices) indicated that correlating errors between items would improve the overall model fit in both groups. Inspection of the modification indices suggested that nine pairs of error-terms should be allowed to correlate: Items 2 and 12, Item 2 and Item parcel 3, Items 16 and 22, Items 16 and 46, Items 39 and 40, Items 44 and 45, Item parcels 2 and 3, Item parcel 7 and Item 23, and Item parcel 9 and Item 38. All the items composing each pair dealt with similar issues, such as intergenerational support, time allocation, and use of electronic devices. Based on empirical (i.e., improved model fit, see Table 7) and theoretical evidence (i.e., theoretical reasoning regarding item content), nine pairs of errorterms were retained in the subsequent analyses.

A series of competing models were tested to investigate the possibility that fewer factors may fit the data better. The competing models were: (1) the Agreement about different areas of relationships and Instrumental aspects of relationship factors were combined, (2) the Emotional intimacy and Instrumental aspects of relationship factors were combined and the Agreement about different areas of relationships factors were dropped, and (3) the Agreement about different areas of relationships and Instrumental aspects of relationship factors were 
dropped. In each of the competing models, the error correlations for nine pairs were retained. Each of these three competing models was compared with the seven-factor model with nine pairs of correlated errors.

Table 7 summarizes the results of the tests of competing models. Among them, the five-factor model fit the data well (see Table 7, Model 5). Interpersonal behaviors such as agreement and conflict regarding financial management and division of household labor may overlap with Emotional intimacy and Marital conflicts factors to a certain extent.

Furthermore, the empirical evidence of high correlations between two factors-Instrumental aspects and Agreement about different areas of relationships - can work against the validity of the Revised MCLI. Thus, I decided to exclude these two factors and its constituent indicators (i.e., items and item parcels), which resulted in omitting 9 items. In sum, the revised CFA model including 19 items and 8 item parcels was used for measurement invariance testing.

In addition to this, the re-estimation process supported allowing Item parcel 5division of household chores - to cross load on the Emotional intimacy and Marital conflicts factors instead of loading on the Instrumental aspects of relationships. Also, the modification indices indicated allowing Item parcels 7, 9 as well as Items 5, 17, 24, and 38 to cross load on other factors besides their primary one. For example, Items 5 and 17, tapping into sexual needs and partner's physical attractiveness were allowed to cross load on the Emotional intimacy factor as well as the Sexual intimacy factor because sexual intimacy may affect emotional climate of relationships or vice versa. Also, Item 38 and Item parcel 9 were similar in their explicit use of the word 'conflict,' which resulted in allowing them to cross load on both Marital conflicts and Intergenerational relationships factors. This series of respecifications and re-estimation resulted in the most improvement in model fit (see Table 7 , 
Model 6). Based on these results, the remainder of the analyses in this study proceeded using the five-factor model with correlated 9 pairs of error-terms and 7 cross-loaded indicators.

Table 7. Model Fits of Competing CFA Models

\begin{tabular}{|c|c|c|c|c|c|}
\hline Model & $\chi^{2}(d f)$ & RMSEA [90\%CI] & CFI & TLI & SRMR \\
\hline \multicolumn{6}{|l|}{1.7 factors } \\
\hline Koreans & $1786.022(506)$ & $0.089[.084, .093]$ & .871 & .857 & .078 \\
\hline Americans & $1441.464(506)$ & $0.072[.068, .077]$ & .879 & .866 & .073 \\
\hline \multicolumn{6}{|c|}{$\begin{array}{l}2.7 \text { factors, nine pairs of } \\
\text { correlated errors }\end{array}$} \\
\hline Koreans & $1425.587(497)$ & $.076[.072, .081]$ & .906 & .894 & .061 \\
\hline Americans & $1274.071(497)$ & $.066[.062, .071]$ & .900 & .887 & .069 \\
\hline \multicolumn{6}{|c|}{$\begin{array}{l}\text { 3. } 6 \text { factors } \\
\text { (combined Agree, and Ins) }\end{array}$} \\
\hline Koreans & $1511.707(503)$ & $.079[.074, .084]$ & .898 & .886 & .064 \\
\hline Americans & $1346.610(503)$ & $.069[.064, .070]$ & .891 & .879 & .072 \\
\hline \multicolumn{6}{|c|}{ 4. 5 factors (combined } \\
\hline \multicolumn{6}{|c|}{ Emo and Ins and dropped } \\
\hline \multicolumn{6}{|l|}{ Agree) } \\
\hline Koreans & $1144.236(386)$ & $.078[.073, .083]$ & .917 & .906 & .065 \\
\hline Americans & $1052.268(386)$ & $.070[.065, .075]$ & .903 & .891 & .072 \\
\hline \multicolumn{6}{|c|}{$\begin{array}{l}5.5 \text { factors } \\
\text { (dropped Agree, and Ins) }\end{array}$} \\
\hline Koreans & $780.014(280)$ & $.074[.068, .081]$ & .936 & .926 & .061 \\
\hline Americans & $681.795(280)$ & $.064[.058, .070]$ & .930 & .919 & .069 \\
\hline \multicolumn{6}{|c|}{$\begin{array}{l}\text { 6. Final model: } 5 \text { factors } \\
\text { (cross-loaded items) }\end{array}$} \\
\hline Koreans & $635.682(298)$ & $.059[.053, .066]$ & .958 & .951 & .037 \\
\hline Americans & $557.400(298)$ & $.050[.043, .056]$ & .957 & .949 & .036 \\
\hline
\end{tabular}

Note. Agree=agreement about different areas of relationships, Ins=instrumental aspects of relationships, Emo=emotional intimacy

\section{Measurement Invariance of Second-Order CFA Models}

The hypothesized second-order CFA model reflected that the positive correlations among the five first-order factors of marital quality (i.e., emotional intimacy, sexual intimacy, marital conflicts, intergenerational relationships, and complaints about partner's lifestyle) are explained by a second-order factor of marital quality (see Figure 2 and 3). Measurement invariance was tested using a series of MG-CFAs with the re-specified model with correlated error-terms and cross-loaded indicators. 
Figure 2. Results of the second-order factor model for Koreans: Unstandardized solution

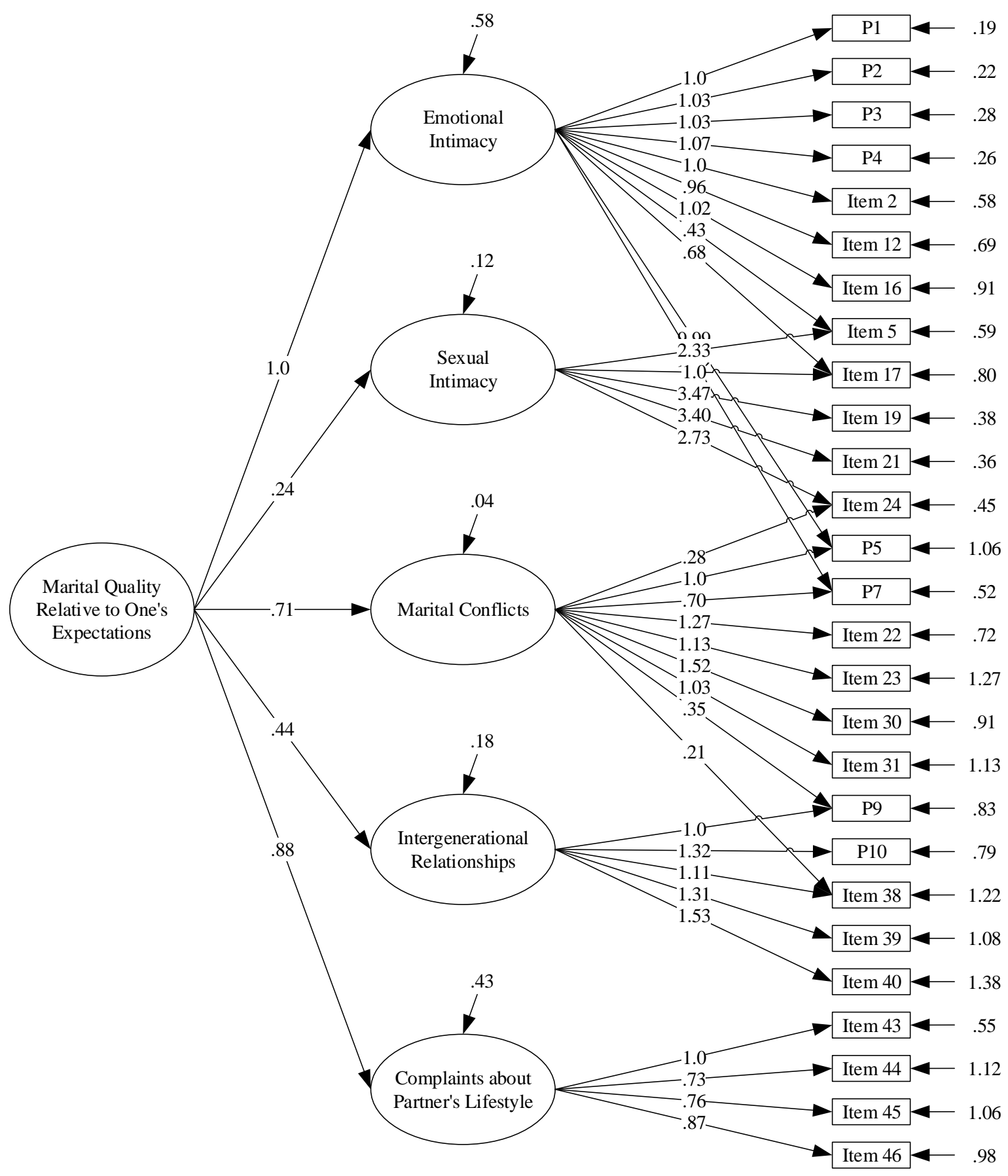

Note. The $R^{2}$ for the first-order factors were emotional climate (.68), sexual intimacy (.38), conflict management (.93), intergenerational exchanges (.57), and complaints about partner's lifestyle (.69). 
Figure 3. Results of the second-order factor model for Americans: Unstandardized solution

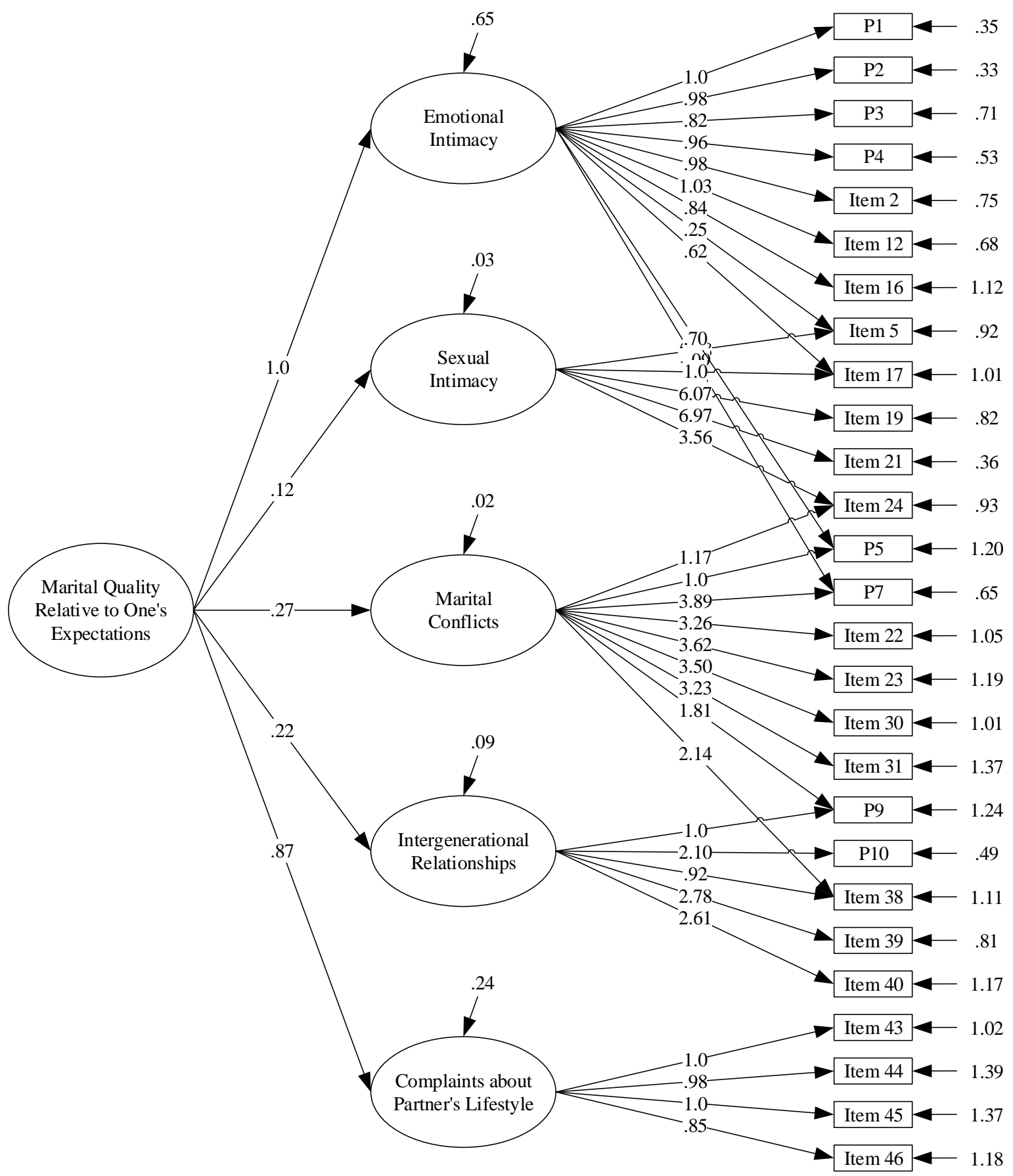

Note. The $R^{2}$ for the first-order factors were emotional climate (.60), sexual intimacy (.32), conflict management (.77), intergenerational exchanges (.34), and complaints about partner's lifestyle (.76).

The first step was to estimate the hypothesized model separately in each group to determine whether the model fit well for Koreans and Americans. For both groups, the model 
fit well (See Table 8). Following the estimation of the model separately with each group, a sequence of nested models was obtained by constraining a set of parameters to be equal across groups (Byrne \& Stewart, 2006; Chen et al., 2005).

Table 8. Model Fits of the Second-order CFA Model

\begin{tabular}{lclllll}
\hline Group & $\chi^{2}(\mathrm{df})$ & RMSEA & 90\% CI for RMSEA & CFI & TLI & SRMR \\
\hline Koreans & $707.846(303)$ & .064 & $.058-.071$ & .950 & .942 & .055 \\
Americans & $605.654(303)$ & .053 & $.047-.059$ & .950 & .942 & .049 \\
\hline
\end{tabular}

Configural invariance (Model 1). In testing for this form of invariance, an unrestricted baseline model was specified in which each group had the same structure. That is, the pattern of fixed and free factor loadings for the first- and second-order factor loadings was constrained to be equal across groups, but different estimates were allowed for the corresponding parameters in the different groups. As can be seen from Table 9 , the $\chi^{2}$ statistic was $1313.500(d f=606), \mathrm{p}<.001$, RMSEA was .05, and CFI was .95. These results indicated an adequate fit of the model to the data.

Invariance of first-order factor loadings (Model 2). In testing for this level of factorial variance, all of the first-order factor loadings were constrained to be equal across groups. This level of invariance was nested within Model1. As can be seen from Table 9, the change of CFI value was not below the cutting score of -.01 for lack of invariance $(\Delta \mathrm{CFI}$ $=.009)$. These results indicated that the first-order factor loadings were invariant across two cultural groups.

Invariance of second-order factor loadings (Model 3). In testing for this level of invariance, all first and second-order factor loadings were constrained to be equal across groups. The CFI value did not decrease, therefore, the second-order factor loadings were invariant across the two groups. 
Invariance of intercepts of measured variables (Model 4). Model 4 was obtained from Model 3 by adding the constraint of equal intercepts of the measured variables across groups. This level of invariance was required to identify potential differences in the intercepts of the measured variables between groups. The results indicated that there was no substantial difference in CFI $(\triangle \mathrm{CFI}=.009)$, indicating that the constraint of invariant item intercepts holds.

Invariance of intercepts of first-order latent factors (Model 5). In testing for this level of invariance, the intercepts of the first-order latent factors were constrained to be equal across groups in addition to intercept of invariance of measured items to compare the secondorder factor means across groups. Model 5 was obtained from Model 4 by adding the constraint of equal first-order factor intercepts across the two groups. Given that there was no substantial difference in CFI (.931 vs. .932), the results indicated that there was no appreciable difference in the intercepts of the first-order factors across two cultures.

Invariance of disturbances of first-order factors (Model 6). In testing for this level of invariance, all first- and second-order factor loadings, the intercepts of the measured variables and the first-order factors, and disturbances of the first-order factors were constrained to be equal across groups. This model was obtained from Model 5 by adding the constraint of equal first-order factor disturbances across groups. The disturbances were considered unique variances that are not shared by the common higher order factor between Korean and American participants. The results indicated that there was no substantial difference in CFI $(\triangle \mathrm{CFI}=.001)$, which indicates that there was no appreciable difference in the disturbances.

Invariance of residual variance of measured variables (Model 7). Model 7 was obtained from Model 6 by adding the constraint of invariant item uniqueness across two 
groups. In other words, all first- and second-order factor loadings, the intercepts of the measured variables and the first-order factors, disturbances of the first-order factors, and residual variances of the measured variables were constrained to be equal across groups. This level of factorial invariance is considered an extremely restrictive test of the data. The change of CFI $(\triangle \mathrm{CFI}=.17)$ indicated that the constraint of invariant item residual variances (item uniqueness) does not hold. Therefore, Model 6, in which the first- and second-order factor loadings, the intercepts of the measured variables and the first-order factors, and the disturbances of the first-order factors were constrained to be equal, represented the highest level of invariance that could fit with these data.

Taken all together, the second-order factor model of marital quality fit the Korean and American sample adequately. The responses to the revised MCLI showed that there was marital quality as a general dimension that underlies the five first-order factors. These findings provide psychometric properties of the revised measure and allow for the testing of differences in factor means across the groups. 
Table 9. Summary of Fit Statistics for Testing Measurement Invariance of Second-order Factor Model

\begin{tabular}{|c|c|c|c|c|c|c|c|}
\hline Model & $\chi^{2}$ & $d f$ & RMSEA & $\begin{array}{l}\text { RMSEA } \\
90 \% \text { CI }\end{array}$ & CFI & $\Delta \mathrm{CFI}$ & TLI \\
\hline \multicolumn{8}{|l|}{ Model 1} \\
\hline Configural invariance & 1313.500 & 606 & .059 & $\begin{array}{l}.054- \\
.063\end{array}$ & .95 & & .94 \\
\hline \multicolumn{8}{|l|}{ Model 2} \\
\hline $\begin{array}{l}\text { First-order factor loadings } \\
\text { invariant }\end{array}$ & 1467.864 & 635 & .062 & $\begin{array}{l}.058- \\
.066\end{array}$ & .941 & .009 & .935 \\
\hline \multicolumn{8}{|l|}{ Model 3} \\
\hline $\begin{array}{l}\text { First- and second-order } \\
\text { factor loadings invariant }\end{array}$ & 1471.537 & 639 & .062 & $\begin{array}{l}.058- \\
.066\end{array}$ & .941 & .000 & .935 \\
\hline \multicolumn{8}{|l|}{ Model 4} \\
\hline $\begin{array}{l}\text { First- and second-order } \\
\text { factor loadings and } \\
\text { intercepts of measured } \\
\text { variables invariant }\end{array}$ & 1625.141 & 665 & .065 & $\begin{array}{l}.061- \\
.069\end{array}$ & .932 & .009 & .928 \\
\hline \multicolumn{8}{|l|}{ Model 5} \\
\hline $\begin{array}{l}\text { First- and second order } \\
\text { factor loadings, and } \\
\text { intercepts of measured } \\
\text { variables and first-order } \\
\text { factors invariant }\end{array}$ & 1638.565 & 666 & .066 & $\begin{array}{l}.062- \\
.070\end{array}$ & .931 & .001 & .928 \\
\hline \multicolumn{8}{|l|}{ Model 6} \\
\hline $\begin{array}{l}\text { First- and second-order } \\
\text { factor loadings, intercepts, } \\
\text { and disturbances of first- } \\
\text { order factors invariant }\end{array}$ & 1664.010 & 671 & .066 & $\begin{array}{l}.062- \\
.070\end{array}$ & .930 & .001 & .927 \\
\hline \multicolumn{8}{|l|}{ Model 7} \\
\hline $\begin{array}{l}\text { First- and second-order } \\
\text { factor loadings, intercepts, } \\
\text { and disturbances of first- } \\
\text { order factors, and residual } \\
\text { variances of measured } \\
\text { variables invariant }\end{array}$ & 1930.500 & 698 & .072 & $\begin{array}{l}.068- \\
.076\end{array}$ & .913 & .017 & .912 \\
\hline
\end{tabular}

\section{Multi-Group Analysis of Similarity in Latent Profile Solutions}

Upon establishing the adequate level of measurement invariance, LPA was used to investigate profile similarity across groups. The first step was to examine whether the same number of profiles could be identified across groups. Because previous research has generally yielded three distinct marital relationship profiles (e.g., Boerner, Jopp, Carr, Sosinsky, \& 
Kim, 2014; Kamp Dush, Taylor, \& Kroeger, 2008), I examined solutions up to four profiles separately in both groups. The fit indices for these solutions are presented in Table 10. Most indices (BIC, SABIC, entropy) supported the three-profile solution in both groups. Based on the results, the three-profile solution was retained for both samples, supporting the configural similarity of the model across Korea and the U.S.

\section{Cross-cultural similarity (structural, dispersion, and distributional) of the}

profiles. A multi-group three-profile model was simultaneously estimated in both samples. I first estimated a model of structural similarity by constraining the within-profile means on the five construct of marital quality to be equal across cultures. Compared with the baseline configural similarity model, the structural similarity model resulted in higher values on the BIC and SABIC, indicating that the within-profile means differ across cultures. Furthermore, a model of distributional similarity resulted in a substantial increase in the value of SABIC, suggesting that the relative size of the profiles differ across cultures (See Table 10). In sum, the results indicated that the three latent profiles can be identified in both groups. However, the nature of the profiles is different across groups. In other words, the profiles have a different meaning across groups. Given that the adequate level of invariance was established in the variable-centered analysis, structural differences may reflect true differences in the nature of the profile themselves. That is, there are cultural differences in terms of the ways in which the each construct of marital quality combines. The discussion section explains these differences to guide for future research. 
Table 10. Fit Statistics from the Latent Profile Analyses

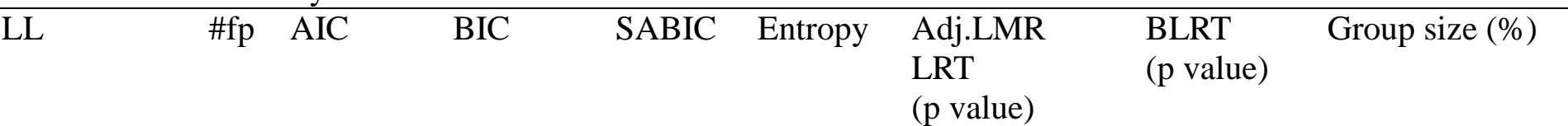

\begin{tabular}{|c|c|c|c|c|c|c|c|c|c|}
\hline & & & & & & & & & \\
\hline $\begin{array}{l}\text { Class enumeration: } \\
\text { Korea }\end{array}$ & & & & & & & & & \\
\hline 1 profile & -2033.367 & 15 & 4096.734 & 4153.352 & 4105.775 & - & - & - & 322 \\
\hline 2 profile & -1944.076 & 26 & 3940.152 & 4038.291 & 3955.822 & .546 & $\begin{array}{l}175.814 \\
(.240)\end{array}$ & $\begin{array}{l}178.582 \\
(.00)\end{array}$ & $\begin{array}{l}\mathrm{C} 1: 202(62 \%) \\
\mathrm{C} 2: 119(37 \%)\end{array}$ \\
\hline 3 profile & -1880.930 & 37 & 3835.859 & 3975.518 & 3858.159 & .736 & $\begin{array}{l}124.335 \\
(.102)\end{array}$ & $\begin{array}{l}126.293 \\
(.00)\end{array}$ & $\begin{array}{l}\mathrm{C} 1: 74(22 \%) \\
\mathrm{C} 2: 107(33 \%) \\
\mathrm{C} 3: 140(43 \%)\end{array}$ \\
\hline 4 profile & $\mathrm{a}$ & - & - & - & - & - & - & - & - \\
\hline $\begin{array}{l}\text { Class enumeration: U.S. } \\
1 \text { profile }\end{array}$ & -2231.977 & 15 & 4493.955 & 4551.994 & 4504.408 & - & - & - & 354 \\
\hline 2 profile & -2131.627 & 26 & 4315.253 & 4415.855 & 4333.372 & .723 & $\begin{array}{l}197.640 \\
(.02)\end{array}$ & $\begin{array}{l}200.701 \\
(.00)\end{array}$ & $\begin{array}{l}\text { C1:206 (58\%) } \\
\text { C2:147 (41\%) }\end{array}$ \\
\hline 3 profile & -2052.469 & 37 & 4178.938 & 4322.102 & 4204.723 & .814 & $\begin{array}{l}155.901 \\
(.248)\end{array}$ & $\begin{array}{l}158.315 \\
(.00)\end{array}$ & $\begin{array}{l}\mathrm{C} 1: 24(6 \%) \\
\mathrm{C} 2: 129(36 \%) \\
\mathrm{C} 3: 200(56 \%)\end{array}$ \\
\hline $\begin{array}{l}4 \text { profile } \\
\text { Cross-cultural similarity }\end{array}$ & $-2019.796^{a b}$ & 48 & - & - & - & - & - & - & \\
\hline Configural & -4404.659 & 70 & 8949.317 & 9265.451 & 9043.194 & .860 & - & - & - \\
\hline Structural (means) & -4455.880 & 55 & 9021.759 & 9270.150 & 9095.519 & .821 & - & - & - \\
\hline $\begin{array}{l}\text { Dispersion (means and } \\
\text { variances) }\end{array}$ & -4480.243 & 40 & 9040.486 & 9221.134 & 9094.130 & .822 & - & - & - \\
\hline $\begin{array}{l}\text { Distributional (means, } \\
\text { variances, probabilities) }\end{array}$ & -4488.558 & 38 & 9053.116 & 9224.731 & 9104.077 & .818 & - & - & - \\
\hline
\end{tabular}


Description of the profiles. The latent profiles from the initial solution are illustrated in Figure 4. In the Korean sample, Profile 1 included $33.5 \%$ of the sample. This profile generally showed a higher level of marital quality with higher scores on each construct of marital quality than other two profiles. Thus, I labeled this profile High-quality Marriages. In Profile 2, all constructs were around average, corresponding to a Middlequality Marriages profile, which was the largest group of all marriages in the Korean sample. Finally, Profile 3 was characterized by all constructs scores that were below average, with the lowest level of sexual intimacy, particularly. Thus, I used the label Low-quality Marriages (characterized as not meeting the needs of the participant) to describe this profile. Profile 3 represented $23 \%$ of the marriages. The three-profile model had an entropy of .736 .

Figure 4. Three-class LPA model for Koreans

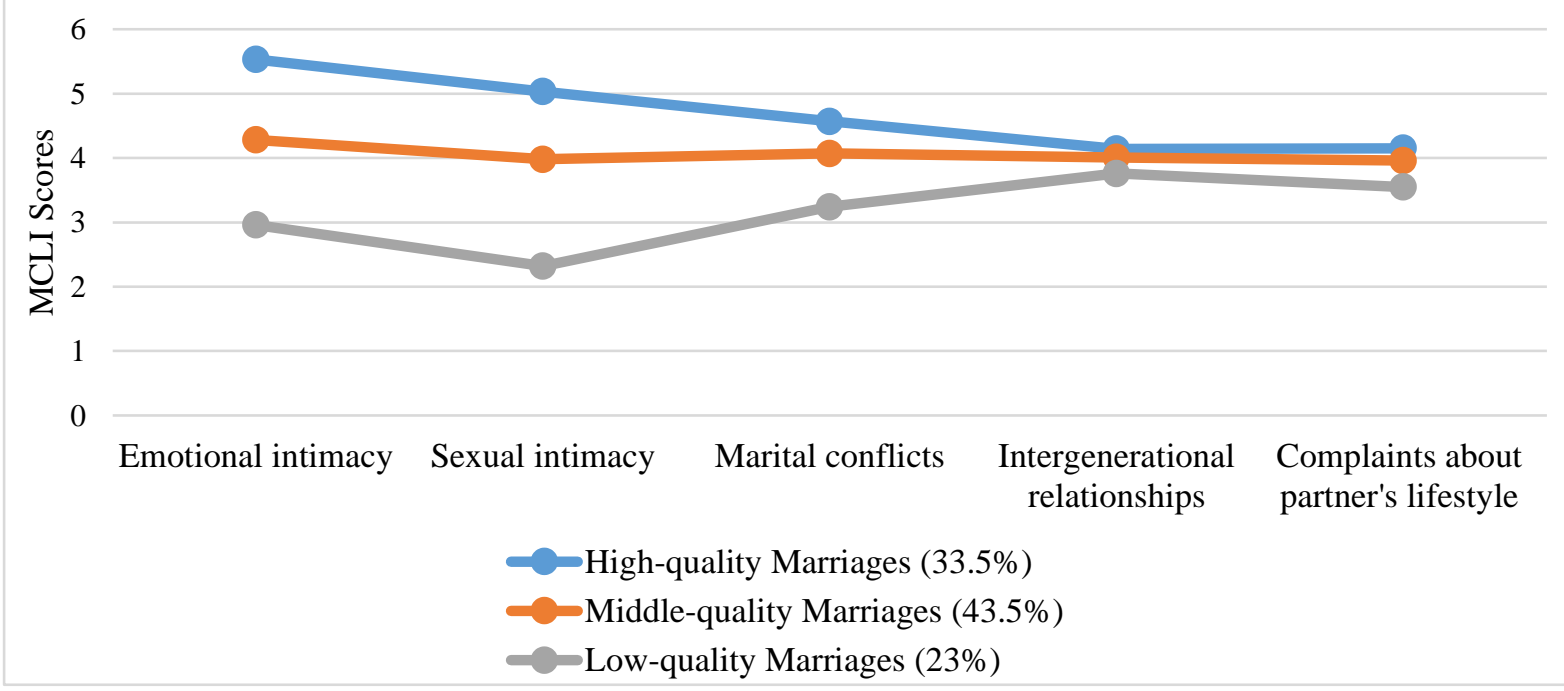

Similarly to the Korean sample, the three-profile solution was retained in the American sample; however, the characteristics of each profile were different (See Figure 5). In Profile 1, all five constructs of marital quality were relatively high and stable compared to the other two Profiles. Thus, I labeled this profile Stable and High-quality Marriages, which was relatively uncommon at $6.8 \%$. Profile 2 was dominated by high levels of Emotional and 
Sexual intimacy, combined with levels of Marital conflicts, Intergenerational relationships, and Complaints about partner's lifestyle that were lower than in Profile 1. It suggested that married adults in this Profile had complaints in these three specific domains of their marriages. Thus, I referred to this profile as High-quality Marriages with Complaints. Lastly, Profile 3 showed low scores on each construct of marital quality, corresponding to a Lowquality Marriages (characterized as not meeting the needs of the participant) profile. It was the largest group and represented $56.5 \%$ of all marriages in the American sample. The threeprofile model yielded a reasonably high level of classification accuracy, with an entropy value of .814

Figure 5. Three-class LPA model for Americans

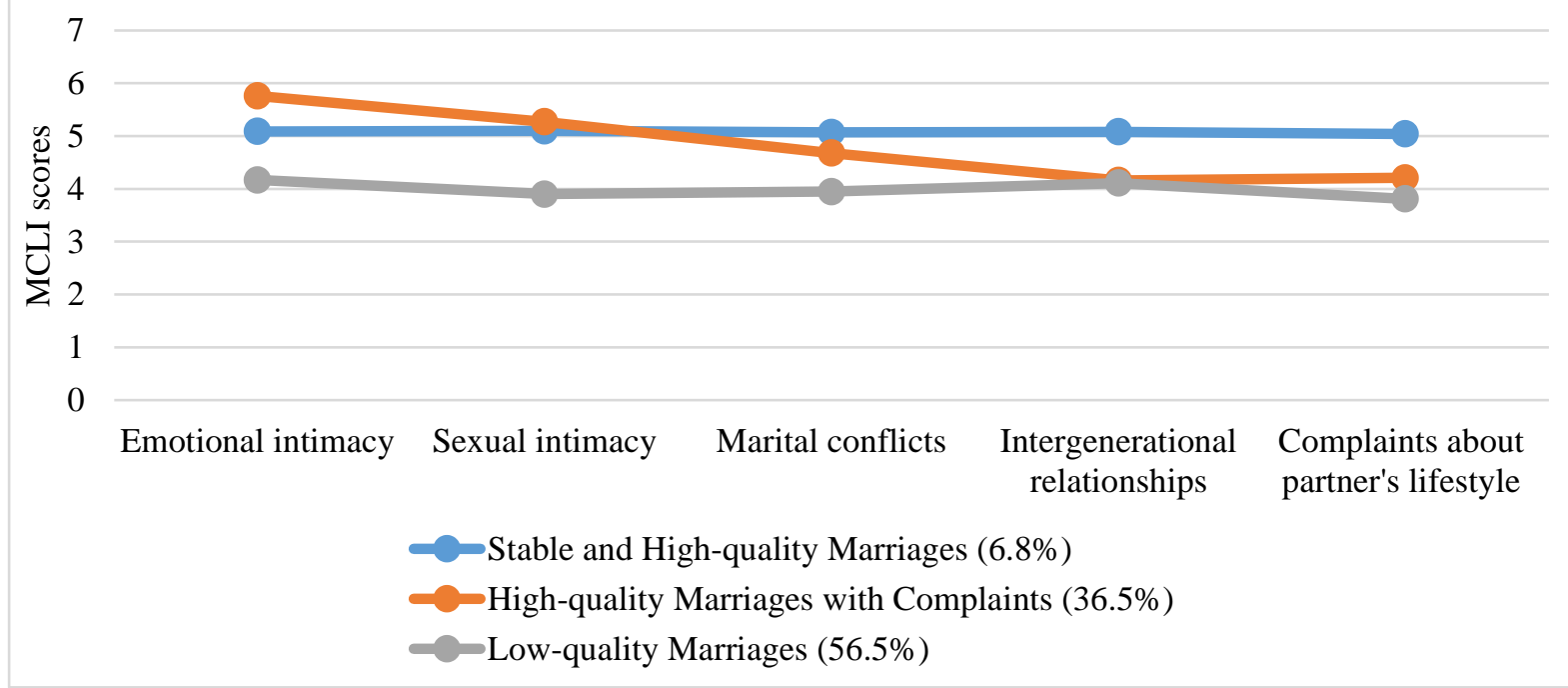

Predictors and outcomes of the profiles. Because of the differences of the withinprofile characteristics (i.e., means and variances) and relative sizes of all profiles across cultures, I added predictors and outcomes to the separate LPA models for the Korean and American sample to explore the relations between profiles and predictors/outcomes. It is important to note that including predictors and outcomes in the models does not change the nature of the profiles. A major objective of including key covariates was to investigate likely 
determinants and consequences of profile membership, which demonstrates construct validity by showing meaningful relations to predictors, correlates, and outcomes (Kam et al., 2016; Morin et al., 2016; Muthén, 2003).

The predictors included in the following analysis were demographic characteristics (i.e., gender, relationship duration, and being a parent). In the Korean sample, as relationship duration increased, the likelihood of membership in Profile 1 (High-quality Marriages) was reduced. Also, being male predicted an increased likelihood of membership into Profile 1 (High-quality Marriages) relative to Profile 3 (Low-quality Marriages). Similarly, being male predicted an increased likelihood of membership into Profile 1 (Stable and High-quality Marriages) relative to Profile 3 (Low-quality Marriages) in the American sample. Being a parent had little implication for profile membership across cultures (See Table 11).

Next, distal outcomes (relationship satisfaction and stability) were added to the LPA models. The mean levels of each outcome in the profiles are reported in Table 12. Levels of relationship satisfaction were significantly greater in Profile 1 (High-quality Marriages) in the Korean sample, supporting the positive relations between MCLI scores and relationship satisfaction across profiles. Similarly, the assessment of outcomes relative to expectations was positively associated with relationship satisfaction in the American sample. Relationship stability was also related to the scores on MCLI, suggesting the positive relations between high-quality marriages and relationship stability regardless of cultural differences. 
Table 11. Results from the Multinomial Logistic Regression Evaluating the Effects of Predictors on Latent Profile Membership

\begin{tabular}{lllllll}
\hline & \multicolumn{2}{l}{ Latent profile 1 vs.2 } & \multicolumn{2}{l}{ Latent profile 1 vs.3 } & \multicolumn{2}{l}{ Latent profile 2 vs.3 } \\
\cline { 2 - 7 } & Coef. (SE) & OR & Coef. (SE) & OR & Coef. (SE) & OR \\
\hline $\begin{array}{l}\text { Korea } \\
\text { Gender (Male) }\end{array}$ & $.518(.386)$ & 1.678 & $.967(.386)^{*}$ & 2.630 & $.449(.331)$ & 1.566 \\
$\begin{array}{l}\text { Relationship } \\
\text { duration }\end{array}$ & $-.066(.022)^{* *}$ & .936 & $-.046(.022)^{*}$ & .955 & $.020(.022)$ & 1.020 \\
$\begin{array}{l}\text { Being a parent } \\
\text { U.S. }\end{array}$ & $-1.454(.793)$ & .233 & $-1.066(.834)$ & .344 & $.388(.425)$ & 1.474 \\
$\begin{array}{l}\text { Gender (Male) } \\
\begin{array}{l}\text { Relationship } \\
\text { duration }\end{array}\end{array}$ & $0.457(.540)$ & 1.579 & $1.035(.520)^{*}$ & 2.815 & $.578(.299)$ & 1.782 \\
$\quad$ Being a parent & $.284(.492)$ & 1.328 & $.631(.485)$ & 1.879 & $.347(.296)$ & 1.414 \\
\hline
\end{tabular}

Note. The coefficients and OR reflect the effects of the predictors on the likelihood of membership into the first listed profile relative to the second listed profile.

Coef=coefficient, $\mathrm{SE}=$ standard error of the coefficient, $\mathrm{OR}=$ odds ratio.

Table 12. Characteristics of the Profiles of Marital Quality on the Outcomes

\begin{tabular}{lcccl}
\hline & Profile 1 & Profile 2 & Profile 3 & Tests of Significance \\
\hline Korea & & & & \\
$\quad$ Relationship satisfaction & 4.138 & 3.376 & 2.401 & $1>2>3$ \\
$\quad$ Relationship stability & 1.839 & 1.677 & 1.457 & $1>2>3$ \\
U.S. & & & & \\
$\quad$ Relationship satisfaction & 4.531 & 4.746 & 3.783 & $1=2>3$ \\
$\quad$ Relationship stability & 1.807 & 1.798 & 1.638 & $1=2>3$ \\
\hline
\end{tabular}

Note. Relationship stability was measured by 5 dichotomous items; however, they were combined and treated as a continuous composite indicator of the construct. 


\section{CHAPTER FIVE: DISCUSSION}

Using the ecological/exchange framework, the MCLI was revised to measure the interpersonal process highlighted by exchange perspectives on the evaluations of relationships for use with Korean married couples. The goals of this study were to examine the factor structure of the revised MCLI and to determine whether the revised measure functioned in the same way across cultures. Broadly speaking, the measure consisted of five dimensions of marital evaluations, which were accounted by a common underlying construct - marital quality. The discussion section begins with an overview of the primary findings. Next, the strengths and limitations of the study are presented. Finally, recommendations and future research directions are provided.

\section{Factor Structure}

The primary goal of the present study was to examine the factor structure of the revised MCLI by conducting a series of CFAs with the Korean and American sample. CFA results indicated a five-factor structure including Emotional intimacy, Sexual intimacy, Marital conflicts, Intergenerational relationships, and Complaints about partner's lifestyle. Each factor consisted of multiple items and item parcels, which were distinct, but related to each other and explained a general marital quality factor.

Based on the literature review and item content analysis, I originally hypothesized that seven constructs compose the MCLI; however, CFA results suggested that two of them —Agreement about different areas of relationships and Instrumental aspects of relationships — do not actually represent different constructs. Moreover, due to the empirical evidence that the Instrumental aspects of relationships factor overlaps with the Emotional intimacy factor, the hypothesized model based on seven constructs was restructured to contain five. In order to make each dimension of the MCLI conceptually clearer, the final 
model included five dimensions that constitute more coherent items closely representing each dimension's core attributes.

Emotional intimacy reflected general happiness and affection towards partner as well as assessments of the relationship such as relationship equality. The present study showed this construct explained a substantial amount of marital quality across cultures. Sexual intimacy was also shown to be a distinct construct of marital quality. The construct of Marital conflicts included agreement or conflicts about certain issues such as division of household labor and the use of leisure time. The present study showed that Marital conflicts explained a substantial amount of variance of marital quality across cultures (i.e., 93\% of variance of marital quality for Koreans and $77 \%$ of variance of marital quality for Americans).

In addition to these three constructs emerged from the original scale, the Intergenerational relationships and Complaints about partner's lifestyle dimensions exhibited distinct constructs of marital quality. Interestingly, the culture-specific aspect of marital relationships - Intergenerational relationships — was shown to be a reliable indicator of marital quality across cultures. This construct tapped into intergenerational exchanges such as emotional and financial support between parents/parents-in-law and married adults. However, the proportion of explained variance in this dimension appeared to be different, namely, $57 \%$ of the variance of marital quality was accounted for by the latent factor of intergenerational relationships for Koreans and marital quality accounted for $34 \%$ of the variance in intergenerational relationships for Americans.

The construct reflecting contemporary marriages, which is Complaints about partner's lifestyle, was also salient across cultures. Although significant changes in marriage occurred over the past years, the "grandparents" of marital assessments (e.g., Locke-Wallace Marital Adjustment Test, Locke \& Wallace, 1959; Dyadic Adjustment Scale, Spanier, 1976) 
have been used with little modification. Thus, the present study took historical changes (e.g., the links between marriage and the workplace and conflict over technology use) into consideration and revealed that these shifts impact marital quality regardless of cultural differences.

In sum, the present study supports the idea that various dimensions of marriage are not combined into a single summary score. Combining the various dimensions-conceptually distinct but empirically correlated — into a single score would lead to obscuring the relations between them. Instead, marital quality should be treated as a general concept that consists of specific marital behaviors and evaluations. Given the fact that all characteristics of marital relationships are not equally important (i.e., some are more salient than others), assessing multiple dimensions of marital quality could provide a detailed picture of how contemporary marriages are experienced and structured within and between cultures.

\section{Generalizability of the Revised MCLI across Cultures}

Measurement invariance examined that the same constructs are measured across different groups. From the finding of configural invariance, I concluded the second-order MCLI structure, as portrayed in Figure 2 and 3, to be functioning equivalently across Korean and American married adults. That is, there was a second-order factor, which is marital quality, that underlies the five first-order factors (i.e., emotional intimacy, sexual intimacy, marital conflicts, intergenerational relationships, and complaints about partner's lifestyle). Results of the measurement invariance testing indicated that the revised MCLI's basic structure and its measurement parameters - including first- and second-order factor loadings, intercepts of measured variables, intercepts of first-order factors, disturbances of first-order factors-were shown to be invariant across cultures.

Evidence of invariant first- and second-order factor loadings reveals the possibility of 
cross-cultural comparisons in the relations between the constructs. Further, invariance of both the intercepts of the measured variables and first-order factors allows us to test the differences in factor means across cultures. In other words, factor mean differences would indicate true cultural differences related to construct bias, which indicates that the construct being measured holds differential meaningfulness across cultures to some extent (van de Vijver \& Tanzer, 2004). Related to this point, the difference between the group mean on the second-order factor can be compared in the case of a hierarchical model, which is the measurement model in the present study. The invariance of disturbances of the first-order factors implies that the unique variance of each first-order factor that is not shared by the second-order factor (i.e., marital quality) is the same. Although the invariance of the uniqueness of the measured variables was not established in the present study, it is important to note that testing for residual invariance is the most advanced level of invariance that is difficult to achieve in cross-cultural research (Byrne \& Campbell, 1999; Byrne \& Watkins, 2003).

As noted above, the second-order factor means in the two cultures were compared, given the adequate level of measurement invariance. The Korean sample was selected as a reference group to obtain an estimate of the difference between the second-order factor means across the two groups and its second-order latent mean was fixed at zero. Invariance of firstand second-order factor loadings and intercepts of the measured variables and first-order factors were constrained to be equal on the Korean and American samples. The result indicated that there was a significant mean difference between the two groups on the secondorder factor $(.32, z=3.66, p<.001)$, suggesting that the American sample had a higher score on the overall marital quality than the Korean counterpart. In sum, the adequate level of measurement invariance provided strong evidence that the measure functions in a similar way across cultures and allowed for comparison of the latent mean difference across groups. 
This study focused on the invariance of the revised MCLI parameters with a variable-centered approach. The primary focus was to examine how the MCLI measures the first and second-order factors included in the MCLI's measurement model across cultures. This approach assumed homogeneity of individuals; in other words, all individuals from the same culture were drawn from a single population. Given that measurement invariance has been established, a logical extension of this measurement invariance research was to address research questions concerned with heterogeneity of individuals within and between cultures with a person-centered approach. This type of research — hybrid variable- and personcentered approaches_-is particularly helpful for psychometric investigations of measurement invariance (Morin et al., 2016). Indeed, when cross-cultural generalizability is examined with the person-centered, the understanding of cross-cultural universality and variation in marital relationships is more nuanced. For this reason, the current study examined groups of individuals with similar profiles of scores across the five first-order factors within cultures, which was further extended to assess generalizability of latent profiles across cultures.

The findings of LPA revealed three distinct groups of married individuals in both cultures, supporting the configural similarity of the profiles. However, the structural, dispersion, and distributional of the profiles appeared to be different. That is, there were group-specific differences, indicating true cultural difference in the nature of the profiles themselves. In the Korean sample, I found that $77 \%$ of the participants were in stable and relatively high-quality marriages and about $23 \%$ were in low-quality marriages. In particular, stable and relatively high-quality marriages were divided into two groups: stable and highquality and stable and middle-quality marriages. Distinguishing high from middle-quality marriages may be useful for future research in terms of understanding the nuanced experiences of happy marriages and their practices. The low-quality marriages were particularly characterized by lower levels of emotional and sexual intimacy in the Korean 
sample. It is worth noting that all three profiles converged in the intergenerational relationship factor; namely, no variability exists in the cultural practices regarding intergenerational exchanges in Korean families. This finding is noteworthy as it suggests that intergenerational relationships might result in strain with spouses even in happy marriages.

In the American sample, the three-profile solution was also retained; however, the nature of the three distinct groups were qualitatively different. I found that about $45 \%$ of the respondents were in high-quality marriages and, among them, there were two different patterns in terms of how the five factors combined as a function of group characteristics. Specifically, $7 \%$ of the participants were in stable high-quality marriages and $36 \%$ were in high-quality marriages with complaints. That is, most participants assigned to the second group held both positive and negative feelings about their relationship. They showed higher levels of emotional and sexual intimacy, but, at the same time, experienced negative exchanges with partners. However, the differences between the profiles did not vary significantly in relationship satisfaction or stability. The results align with the idea that even happy marriages have both positive and negative attributes, and, therefore, conceptualization and operationalization of marital quality should reflect both aspects (Kiecolt-Glaser \& Newton, 2001). Furthermore, it also indicates that the amount of conflict itself does not necessarily impact marital quality in a negative way. While the third profile in the American sample showed a relatively low level of marital quality across all five dimensions, this profile appeared to be similar to the Middle-quality Marriages profile in the Korean sample.

Such separate LPA models were further examined with predictors (i.e., gender, relationship duration, being a parent) and outcomes (i.e., relationship satisfaction and stability) to test the relations between profiles and predictors/outcomes. Results provided evidence for predictive similarity in gender, suggesting that the relation between gender (i.e., 
being male) and membership in higher quality marriages was similar across cultures. Further, the relations between profile membership and outcomes provided evidence for the construct validity of the revised MCLI, indicating that profile membership (i.e., stable and higher quality marriages) was related to relationship satisfaction and stability across cultures.

Taken together, an observation of cross-cultural mean score difference is interesting because the observation of cross-cultural differences would be the vehicle for further research, such as addressing systematic patterns in cross-cultural differences. However, as cross-cultural research has moved forward from a mere demonstration of cross-cultural differences to the interpretation of these differences, the results of the LPA analyses go beyond the exploration of averages of the groups and investigate profile similarity and difference across groups. Clearly, the testing of measurement invariance indicates that the Korean and U.S. cultures do not differ significantly with regard to the construct of marital quality; however, individuals from different cultures exhibit different profiles, suggesting the different patterns in various dimensions of marital quality between cultures. Thus, the present study serves as an example of full-fledged cross-cultural research in the sense that the findings make not only a contribution to the observation of cross-cultural difference, but have the potential to look for a valid explanation such as causes and patterns of these differences in marital quality across cultures, as well.

\section{Limitations}

The primary purpose of the study was to explore the key constructs of contemporary marriages and the relations between the constructs across cultures, rather than identify individual items and their relations to constructs. In this regard, parceling was used to minimize the specific variances of each item and to make a more parsimonious representation of the constructs. It has potential advantages when the intent of the research is to understand 
the nature of the construct and its relations to other constructs. However, it can be argued that using parcels may have undesired effects on measurement invariance testing (Nasser \& Wisenbaker, 2003). For example, Meade and Kroustalis (2006) showed that CFA models having parcels were more likely to show measurement invariance erroneously than models using individual items as indicators of latent factors. On the other hand, Little and colleagues (2013) argued that parceling has benefits in the context of invariance testing because the unique variances of items are reduced and error variance is negligible relative to true-score variance of items. Due to these benefits of parceling, the present study employed a theorydriven parceling strategy as opposed to selecting items randomly, which in turn made testing its invariance across groups more meaningful. However, it is worth noting that the present study cannot be justified in concluding that the individual items on the MCLI are invariant across cultures because of the use of the item parcels. Future measurement work should look further at more knowledgeable and efficient application of parcels and the role of item parceling in the context of invariance testing.

An additional limitation concerns the unit of the analyses. From a theoretical point of view, the ecological/exchange framework assumes that each partner is influenced by the partner's experiences and expectations of the relationships. In other words, it is important to use dyadic data collected from both partners' views on exchange processes. However, due to a lack of time and resources, this study could only recruit married individuals, instead of couples. Given that the present study was based on individuals' reports of their experiences in relationships, future research is necessary using dyadic data in order to obtain a more comprehensive understanding of exchange relationships within and between cultures. This would allow us to examine the diverse characteristics of different groups of couples, indicating gender differences in each dimension of the MCLI. 
Despite these limitations, this study contributes to the understandings of crosscultural similarities and differences of marital relationships, which in turn raises awareness to a need to integrate cultural factors into the measurement of marriage and family constructs. While international scholars have used U.S.-based measures for their studies of marital and family issues without questioning the applicability of the measures outside of the U.S., this study revised the MCLI in a way that is culturally sensitive while being conceptually grounded and psychometrically sound, providing strong evidence that the revised MCLI measures the same factors in the same way across cultures, which allowed for meaningful group comparisons. In addition, this study moved beyond assuming homogeneity of the samples and instead explored differences and similarities of subgroups of participants within and between cultures. In doing so, it provided better understandings of the variability and uniqueness that exists within relationships across cultures.

\section{Future Research Directions}

This study specifically focused on the invariance of the revised MCLI across two cultural groups, Korea and the U.S. A logical extension of this measurement invariance research would be to replicate these analyses with diverse samples for generalizability. That is, future studies should be conducted to provide richer implications for each dimension of the MCLI across married individuals from diverse backgrounds (e.g., different cultural/ethnic groups as well as sexual orientation). International scholars in relationship research are encouraged to employ the revised MCLI in order to better understand and evaluate relationship dynamics in different sociocultural contexts. Such future studies would open new directions for understanding the role of contextual factors in the psychometric properties of marital measures.

In addition, a criterion for judging the model fit in second-order factor models should 
be discussed in future studies. The frequently used criteria was changes in CFI and chi-square difference statistics in previous literature. For example, researchers used changes in CFI (e.g., Byrne \& Stewart, 2006; Chen et al., 2005), chi-square difference (e.g., Dimitrov, 2010), and Satorra-Bentler scaled chi-square difference (e.g., Byrne \& Stewart, 2006) as a primary evaluative strategy. However, the current study did not use the chi-square value because of its sensitivity to sample size (Cheung \& Rensvold, 2002). Given a lack of complete understanding of the performance of fit indexes in measurement invariance testing in secondorder models, additional work will be required to determine the extent to which measurement invariance holds across groups.

\section{Conclusion}

Despite repeated calls for cross-cultural research in measurement, researchers in countries outside of the U.S. rarely question the use of U.S.-based measures for their studies of marital and family issues. However, cultural values influence family themes, customs, rules, and how marriages are structured and experienced. Thus, measures need to be adjusted to reflect these different cultural orientations. In this regard, developing instruments that are culturally sensitive while being conceptually grounded and psychometrically sound is important in order for research on couples and families in countries outside the U.S., and in the instance of my study, in Asian countries, to go forward.

The present study provides an example of studying the psychometric properties of U.S. based measures being used in Asian cultures through a cultural lens. The results indicate that the revised MCLI holds measurement invariance-whether the measure reflects the same factors in the same way across cultures — which in turn provides the possibility for meaningful group comparisons. Additionally, this study moves the literature beyond just assuming that individuals from the same culture form one homogenous group, to instead 
understanding the variability observed within them. In doing so, it reveals how each dimension of marital quality plays a role in making up the different subgroups of married adults within and between cultures. Given the context of living in a multicultural environment that impacts what we research and how we practice, understandings of family dynamics in different sociocultural contexts with a proper measurement can contribute the advancement of marital measurement. Finally, this study may serve as an impetus for international scholarship that could promote cultural and racial diversity in relationship research. 


\section{References}

Amato, P. R., Johnson, D. R., Booth, A., \& Rogers, S. J. (2007). Alone together: How marriage in America is changing. Cambridge, MA: Harvard University Press.

Attila, P., András, I., Tünde, S., \& Péter, T. (2014). Psychometric characteristics of the Hungarian version of the Marital Comparison Level Index. Mentálhigiéné És Pszichoszomatika, 15(3), 215-228.

Bianchi, S. M. \& Milkie, M. A. (2010), Work and family research in the first decade of the 21st century. Journal of Marriage and Family, 72, 705-725. doi:10.1111/j.17413737.2010.00726.x

Boerner, K., Jopp, D. S., Carr, D., Sosinsky, L., \& Kim, S.-K. (2014). "His" and "Her" Marriage? The role of positive and negative marital characteristics in global marital satisfaction among older adults. The Journals of Gerontology Series B: Psychological Sciences and Social Sciences, 69(4), 579-589. http://doi.org/10.1093/geronb/gbu032

Booth, A., Johnson, D., \& Edwards, J. N. (1983). Measuring marital instability. Journal of Marriage and the Family, 45(2), 387-394. http://dx.doi.org/10.2307/351516

Brislin, R. W. (1986). The wording and translation of research instruments. In W. J. Lonner \& J. W. Berry (Eds.), Field methods in cross-cultural research (pp. 137-164). Beverly Hills, CA: Sage.

Brown, T. A. (2006). Confirmatory factor analysis for applied research. New York, NY: Guilford Publications.

Buhrmester, M., Kwang, T., \& Gosling, S. D. (2011). Amazon's Mechanical Turk: A new source of inexpensive, yet high-quality, data? Perspectives on psychological science, 
6(1), 3-5. https://doi.org/10.1177\%2F1745691610393980

Burns, T. (1973). A structural theory of social exchange. Acta Sociologica, 16(3), 188-208.

Byrne, B. M., \& Campbell, T. L. (1999). Cross-cultural comparisons and the presumption of equivalent measurement and theoretical structure. A look beneath the surface. Journal of Cross-Cultural Psychology, 30(5), 555-574.

Byrne, B. M., Shavelson, R. J., \& Muthén, B. (1989). Testing for the equivalence of factor covariance and mean structures: The issue of partial measurement invariance. Psychological bulletin, 105(3), 456-466.

Byrne, B. M., \& Stewart, S. M. (2006). Teacher's corner: The MACS approach to testing for multigroup invariance of a second-order structure: A walk through the process. Structural Equation Modeling: A Multidisciplinary Journal, 13(2), 287-321, doi: 10.1207/s15328007sem1302_7

Byrne, B. M., \& van de Vijver, F. J. R. (2014). Factorial structure of the family values scale from a multilevel-multicultural perspective. International Journal of Testing, 14(2), 168-192. https://doi.org/10.1080/15305058.2013.870903

Byrne, B. M., \& Watkins, D. (2003). The issue of measurement invariance revisited. Journal of Cross-Cultural Psychology, 34(2), 155-175. https://doi.org/10.1177/0022022102250225

Carter, N. T., Dalal, D. K., Lake, C. J., Lin, B. C., \& Zickar, M. J. (2011). Using mixed-model item response theory to analyze organizational survey responses: An illustration using the job descriptive index. Organizational Research Methods, 14(1), 116-146. https://doi.org/10.1177\%2F1094428110363309

Chan, Y-H., \& Rudowicz, E. (2002). The Chinese version of the Marital Comparison Level 
Index revisited. Psychological Reports, 91(3f), 1143-1147.

Chen, F. F., Sousa, K. H., \& West, S. G. (2005). Teacher's corner: Testing measurement invariance of second-order factor models. Structural Equation Modeling: A Multidisciplinary Journal, 12(3), 471-492. https://doi.org/10.1207/s15328007sem1203_7

Chesley, N. (2005). Blurring boundaries? Linking technology use, spillover, individual distress, and family satisfaction. Journal of Marriage and Family, 67, 1237-1248. doi:10.1111/j.1741-3737.2005.00213.x

Cheung, G. W., \& Rensvold, R. B. (2002). Evaluating goodness-of-fit indexes for testing measurement invariance. Structural Equation Modeling: A Multidisciplinary Journal, 9(2), 233-255. https://doi.org/10.1207/S15328007SEM0902_5

Cheung, F. M., van de Vijver, F. J. R., \& Leong, F. T. L. (2011). Toward a new approach to the study of personality in culture. American Psychologist, 66(7), 593-603. http://psycnet.apa.org/doi/10.1037/a0022389

Choi, S. H., \& Han, M.S. (2011). A study on the relation between the conflict, interpersonal relation, self-efficacy, and life satisfaction of parents-in-law living together with daughter-in-law. Society of Korean Thought and Culture, 59, 391-415.

Chung, H. (2004). Application and revision of the Kansas marital satisfaction scale for use with Korean couples. Psychological Reports, 95(3), 1015-1022.

Claxton, A., \& Perry-Jenkins, M. (2008). No fun anymore: Leisure and marital quality across the transition to parenthood. Journal of Marriage and Family, 70, 28-43. doi:10.1111/j.1741-3737.2007.00459.x 
Coyne, S. M., Stockdale, L., Busby, D., Iverson, B., \& Grant, D. M. (2011). "I Luv U :)!": A descriptive study of the media use of individuals in romantic relationships. Family Relations, 60(2), 150-162. https://doi.org/10.1111/j.1741-3729.2010.00639.x

Dimitrov, D. M. (2010). Testing for factorial invariance in the context of construct validation. Measurement and Evaluation in Counseling and Development, 43(2), 121-149. https://doi.org/10.1177\%2F0748175610373459

Eriksson, K., \& Simpson, B. (2010). Emotional reactions to losing explain gender differences in entering a risky lottery. Judgment and Decision Making, 5, 159-163.

Flood, S. M., \& Genadek, K. R. (2016). Time for each other: Work and family constraints among couples. Family Relations, 78, 142-164. doi:10.1111/jomf.12255

Gager, C., \& Sanchez, L. (2003). Couples' perceptions of time spent together, marital quality, and the risk of divorce. Journal of Family Issue, 24(1), 21-50. https://doi.org/10.1177\%2F0192513X02238519

Gosling, S. D., Vazire, S., Srivastava, S., \& John, O. P. (2004). Should we trust web-based studies? A comparative analysis of six preconceptions about internet questionnaires. American psychologist, 59(2), 93-104.

Horn, J. L., \& McArdle, J. J. (1992). A practical and theoretical guide to measurement invariance in aging research. Experimental aging research, 18(3), 117-144.

Kam, C., Morin, A. J., Meyer, J. P., \& Topolnytsky, L. (2016). Are commitment profiles stable and predictable? A latent transition analysis. Journal of Management, 42(6), 1462-1490. https://doi.org/10.1177\%2F0149206313503010

Kamp Dush, C. M., Taylor, M. G., \& Kroeger, R. A. (2008). Marital happiness and 
psychological well-being across the life course. Family Relations, 57, 211-226. doi:10.1111/j.1741-3729.2008.00495.x

Kaplan, D. (1989). Model modification in covariance structure analysis: Application of the expected parameter change statistic. Multivariate Behavioral Research, 24(3), 285305.

Kiecolt-Glaser J. K., \& Newton, T. L. (2001). Marriage and health: His and hers. Psychological Bulletin, 127(4), 472-503. http://psycnet.apa.org/doi/10.1037/00332909.127.4.472

Kim, K., Zarit, S. H., Fingerman, K. L., \& Han, G. (2015). Intergenerational exchanges of middle-aged adults with their parents and parents-in-law in Korea. Journal of Marriage and Family, 77(3), 791-805. doi:10.1111/jomf.12185

Kline, R. B. (2011). Principles and practice of structural equation modeling. New York, NY: Guilford press.

Kwon, J.-H., \& Choi, K.-M. (1999). A validation study of the Korean Marital Satisfaction Inventory. The Korean Journal of Clinical Psychology, 18(1), 123-139.

Lavee, Y., \& Ben-Ari, A. (2007). Relationship of dyadic closeness with work-related stress: A daily diary study. Journal of Marriage and Family, 69, 1021-1035. doi:10.1111/j.1741-3737.2007.00428.x

Lenhart, A., \& Duggan, M. (2014, February, 11). Couples, the internet, and social media. Pew Research Center. Retrieved from http://www.pewinternet.org/2014/02/11/couples-the-internet-and-social-media/

Little, T. D. (1997). Mean and Covariance Structures (MACS) analyses of cross-cultural data: Practical and theoretical issues. Multivariate Behavioral Research, 32(1), 53-76. 
Little, T. D., Cunningham, W. A., Shahar, G., \& Widaman, K. F. (2002). To parcel or not to parcel: Exploring the question, weighing the merits. Structural Equation Modeling, 9(2), 151-173. http://dx.doi.org/10.1207/S15328007SEM0902_1

Little, T. D., Rhemtulla, M., Gibson, K., \& Schoemann, A. M. (2013). Why the items versus parcels controversy needn't be one. Psychological Methods, 18(3), 285-300. doi:10.1037/a0033266

Lo, Y., Mendell, N. R., \& Rubin, D. B. (2001). Testing the number of components in a normal mixture. Biometrika, 88, 767-778. https://doi.org/10.1093/biomet/88.3.767

Locke, H. J., \& Wallace, K. M. (1959). Short marital-adjustment and prediction tests: Their reliability and validity. Marriage and Family Living, 21(3), 251-255.

Madathil, J., \& Benshoff, J. M. (2008). Importance of marital characteristics and marital satisfaction: A comparison of Asian Indians in arranged marriages and Americans in marriages of choice. The Family Journal, 16(3), 222-230. https://doi.org/10.1177\%2F1066480708317504

Marriage and Divorce. (n.d.). Retrieved from https://www.cdc.gov/nchs/fastats/marriagedivorce.htm

Marriage and Divorce Statistics in 2016. (2017, March 22). Retrieved from http://kostat.go.kr/portal/eng/pressReleases/1/index.board?bmode=read\&bSeq=\&aSeq $=360678 \&$ page $\mathrm{No}=1 \&$ row Num $=10 \&$ navCount $=10 \&$ currPg $=\&$ sTarget $=$ title $\&$ sTxt $=\mathrm{di}$ vorcea

Mazmanian, M. A., Orlikowski, W. J., \& Yates, J. (2005). Crackberries: The social implications of ubiquitous wireless e-mail devices. In C. Sørensen, Y. Yoo, K. 
Lyytinen, \& J. DeGross (Eds.), Designing ubiquitous information environments: Socio-technical issues and challenges (pp. 337-343). New York, NY: Springer.

McCoach, B., Gable, R. K., \& Madura, J. P. (2013). Instrument development in the affective domain: School and corporate applications. New York, NY: Springer.

McDaniel, B. T., \& Coyne, S. M. (2016). "Technoference”: The interference of technology in couple relationships and implications for women's personal and relational wellbeing. Psychology of Popular Media Culture, 5(1), 85-98. http://dx.doi.org/10.1037/ppm0000065

McDonald, G. W. (1981). Structural Exchange and Marital Interaction. Journal of Marriage and Family, 43(4), 825-839.

Meade, A. W., \& Kroustalis, C. M. (2006). Problems with item parceling for confirmatory factor analytic tests of measurement invariance. Organizational Research Methods, 9, 369-403. https://doi.org/10.1177\%2F1094428105283384

Meredith, W. (1993). Measurement invariance, factor analysis and factorial invariance. Psychometrika, 58(4), 525-543.

Morin, A. J., Meyer, J. P., Creusier, J., \& Biétry, F. (2016). Multiple-group analysis of similarity in latent profile solutions. Organizational Research Methods, 19(2), 231254. https://doi.org/10.1177\%2F1094428115621148

Morr Serewicz, M. C. (2006). The difficulties of in-law relationships. In D. C. Kirkpatrick, S. Duck, \& M. K. Foley (Eds.), Relating difficulty: The process of constructing and managing difficult interaction (pp. 101-118). Mahwah, NJ: Erlbaum.

Muthén, B. (2003). Statistical and substantive checking in growth mixture modeling: Comment on Bauer and Curran (2003). Psychological Methods, 8(3), 369-377. 
http://dx.doi.org/10.1037/1082-989X.8.3.369

Muthén, L.K. \& Muthén, B.O. (2017). Mplus user's guide (8th ed.). Los Angeles, CA: Muthén \& Muthén.

Nasser, F., \& Wisenbaker, J. (2003). A Monte Carlo study investigating the impact of item parceling on measures of fit in confirmatory factor analysis. Structural Equation Modeling: A Multidisciplinary Journal, 13(2), 204-228. doi: $10.1207 / \mathrm{s} 15328007 \mathrm{sem} 1302 \_3$

Nearly $100 \%$ of Households in South Korea Now Have Internet Access, Thanks to Seniors (2017, Jan). Retrieved from https://www.forbes.com/sites/elaineramirez/2017/01/31/nearly-100-of-households-insouth-korea-now-have-internet-access-thanks-to-seniors/\#7be0d87b5572

Nye, F. I. (1979). Choice, exchange and the family. In W. Burr, R. Hill, F. I. Nye, \& I. Reiss (Eds.), Contemporary theories about the family (Vol. 2, pp. 1-41). New York, NY: Free Press.

Nylund, K.L., Asparouhov, T. \& Muthén, B.O. (2007). Deciding on the number of classes in latent class analysis and growth mixture modeling: A Monte Carlo simulation study. Structural Equation Modeling: A Multidisciplinary Journal, 14(4), 535-569, doi: $10.1080 / 10705510701575396$

OECD (2017), Education at a Glance 2017: OECD Indicators, OECD Publishing, Paris, https://doi.org/10.1787/eag-2017-en.

OECD (2018), Fertility rates (indicator). doi: 10.1787/8272fb01-en.

OECD (2018), Hours worked. doi: 10.1787/47be1c78-en (2018). 
OECD (2018), Population with tertiary education. doi: 10.1787/0b8f90e9-en

Ok, S.W. (2011). Continuity and change in patrilineal culture of Korean families. In Korean Family Studies Association (Ed.), Korean families: Continuity and change (pp. 1-18). Seoul, Korea: Seoul National University Press.

Paolacci, G., \& Chandler, J. (2014). Inside the turk: Understanding mechanical turk as a participant pool. Current Directions in Psychological Science, 23(3), 184-188. https://doi.org/10.1177\%2F0963721414531598

Park, S. Y., \& Cho, S. H. (2010). Factors contributing to conflicts between daughters-in-law and parents-in-law. Korean Journal of Family Welfare, 15(3), 5-26.

Park, N., Lee, S., \& Chung, J. E. (2016). Uses of cellphone texting: An integration of motivations, usage patterns, and psychological outcomes. Computers in Human Behavior, 62, 712-719. https://doi.org/10.1016/j.chb.2016.04.041

Rappa, A. L., \& Tan, S-H. (2003). Political implications of Confucian familism. Asian Philosophy, 13, 87-102.

Record shares of Americans now own smartphones, have home broadband. (2017, January). Retrieved from http://www.pewresearch.org/fact-tank/2017/01/12/evolution-oftechnology/

Roberts, J. A., \& David, M. E. (2016). My life has become a major distraction from my cell phone: Partner phubbing and relationship satisfaction among romantic partners. Computers in Human Behavior, 54, 134-141. https://doi.org/10.1016/j.chb.2015.07.058

Roberts, N. A., \& Levenson, R. W. (2001). The remains of the workday: Impact of job stress and exhaustion on marital interaction in police couples. Journal of Marriage and 
Family, 63,1052-1067. doi:10.1111/j.1741-3737.2001.01052.x

Roxburgh, S. (2006). "I wish we had more time to spend together..." The distribution and predictors of perceived family time pressures among married men and women in the paid labor force. Journal of Family Issues, 27(4), 529-553.

https://doi.org/10.1177\%2F0192513X05284008

Sabatelli, R. M. (1984). The Marital Comparison Level Index: A measure for assessing outcomes relative to expectations. Journal of Marriage and Family, 46(3), 651-662.

Sabatelli, R. M. (1999). Marital commitment and family life transitions: A social exchange perspective on the construction and deconstruction of intimate relationships. In J. M. Adams \& W. H. Jones (Eds.), Handbook of Interpersonal Commitment and Relationship Stability. New York, NY: Kluwer Academic/Plenum Publishers.

Sabatelli, R. M., \& Bartle-Haring, S. (2003). Family-of-origin experiences and adjustment in married couples. Journal of Marriage and Family, 65(1), 159-169.

Sabatelli, R. M., Lee, H., \& Ripoll-Núñez, K. (2018), Placing the Social Exchange Framework in an Ecological Context. Journal of Family Theory \& Review, 10, 32-48. doi:10.1111/jftr.12254

Sabatelli, R.M., \& Ripoll-Núñez, K. (2003). Variations in marriage over time: An ecological/exchange theory. In M. Coleman \& L. Ganong (Eds.), Handbook of Contemporary Families (pp.79-95). Thousand Oaks, CA: Sage.

Sabatelli, R. M., \& Shehan, C. L. (1993). Exchange and resource theories. In P. G. Boss \& W. J. Doherty \& R. LaRossa \& W. R. Schumm \& S. K. Steinmetz (Eds.), Sourcebook of Family Theories and Methods: A contextual approach (pp. 385-411). New York, NY: Plenum Press. 
Schmitt, N., \& Kuljanin, G. (2008). Measurement invariance: Review of practice and implications. Human Resource Management Review, 18(4), 210-222. doi: 10.1016/j.hrmr.2008.03.003

Shek, D. T. L., Lam, M. C., Tsoi, K. W., \& Lam, C. M. (1993). Psychometric properties of the Chinese version of the Kansas Marital Satisfaction Scale. Social Behavior and Personality: An International journal, 21(3), 241-249.

Snyder, D. K. (1997). Manual for the Marital Satisfaction Inventory-Revised (MSI-R). Los Angeles, CA: Western Psychological Services.

Spanier, G. B. (1976). Measuring dyadic adjustment: New scales for assessing the quality of marriage and similar dyads. Journal of Marriage and the Family, 38(1), 15-28.

Statistics Korea. (2017). 2016 report on the social survey (Publication No. 11-1240000000165-10). Daejeon, Korea: Statistics Korea. Retrieved from http://kosis.kr/upsHtml/online/downSrvcFile.do?PUBCODE=KN\&SEQ=1741\&FILE _NAME=1101.pdf

Steenkamp, J. B. E., \& Baumgartner, H. (1998). Assessing measurement invariance in crossnational consumer research. Journal of consumer research, 25(1), 78-90. https://doi.org/10.1086/209528

10 Facts about Smartphones as the iPhone turns 10. (2017, June). Retrieved from http://www.pewresearch.org/fact-tank/2017/06/28/10-facts-about-smartphones/

Thibaut, J. W., \& Kelley, H. H. (1959). The social psychology of groups. New York, NY : Wiley. 
Tofighi, D., \& Enders, C. K. (2008). Identifying the correct number of classes in a growth mixture model. In Hancock, G. R., \& Samuelsen, K. M. (Eds.), Advances in latent variable mixture models (pp. 317-341). Charlotte, NC: Information Age.

Vandenberg, R. J., \& Lance, C. E. (2000). A review and synthesis of the measurement invariance literature: Suggestions, practices, and recommendations for organizational research. Organizational Research Methods, 3(1), 4-70. https://doi.org/10.1177\%2F109442810031002

Van de Vijver, F., \& Tanzer, N. K. (2004). Bias and equivalence in cross-cultural assessment: An overview. European Review of Applied Psychology, 54(2), 119-135.

Wu, A. D., Li, Z., \& Zumbo, B. D. (2007). Decoding the meaning of factorial invariance and updating the practice of multi-group confirmatory factor analysis: A demonstration with TIMSS data. Practical Assessment, Research \& Evaluation, 12(3), 1-26.

Yang, H.-C. (2004). The effects of gender role ideology, wife's employment, and perceived equity on marital quality: A case of young middle-class couples in Korea. (Unpublished doctoral dissertation). The University of Connecticut, United States, Connecticut.

Yoo, Y., Cho, H., \& Kwon, J. (2008). Effects of family-of-origin characteristic and conflicts with in-Laws on marital satisfaction. Korean Journal of Psychology: Women, 13(4), 433-451.

Yu, W. (2015). Placing families in context: Challenges for cross-national family research. Journal of Marriage and Family, 77, 23-39. doi:10.1111/jomf.12152 


\section{Appendix}

\section{Information Sheet for Participation in a Research Project \\ University of Connecticut}

Principal Investigator: Ronald. M. Sabatelli, Ph.D.

Student Investigator: Hyanghee Lee, M.A.

Study title: Toward a Culturally Robust Measure to Assess Marital Relationships for Koreans Invitation to participate

You are invited to participate in a study that I am conducting for my dissertation at the University of Connecticut. This information sheet explains the purpose of the study and provides a description of your involvement and rights as a participant. Please review this information sheet carefully and ask any questions about the study at any time.

\section{$\underline{\text { Purpose }}$}

The primary focus of this study is to develop a culturally relevant and robust measure to assess marital quality to be used with Asian couples, with a particular emphasis on Korean couples.

\section{Description of Procedures}

If you decide to participate in this study, you will be instructed on how to complete an on-line survey questionnaire. The survey includes questions designed to elicit information about you, including demographic information, information about your experiences in your family of origin, and information about your experiences in your current intimate relationship. It will take approximately 20 minutes to complete the questionnaire. Your participation in my dissertation research project is completely voluntary. All information that you provide will be anonymous and confidential - that is, no personal identifying information will be collected and there is no way for any personal information to be connected to your responses on the questionnaire. Your participation in this project makes you eligible to receive $\$ 2$ after completing the questionnaire.

\section{Contacts}

If you have further questions about this project or if you have a research-related problem, you can contact the student investigator (Hyanghee Lee, Hyanghee.lee@uconn.edu) or faculty investigator (Dr. Sabatelli, Ronald.sabatelli@uconn.edu). If you have any questions concerning your rights as a research participant, you can contact the University of Connecticut Institutional Review Board (IRB) at 860-486-8802. 
Are you currently married?

$\circ$ Yes

$\circ$ No

The following are statements about your current marital relationship in a general sense. Please respond to them by indicating how satisfied you are.

\begin{tabular}{l|ccccc} 
& $\begin{array}{l}\text { Extremely } \\
\text { dissatisfied }\end{array}$ & $\begin{array}{c}\text { Somewhat } \\
\text { dissatisfied }\end{array}$ & $\begin{array}{c}\text { Neither } \\
\text { dissatisfied } \\
\text { nor } \\
\text { satisfied }\end{array}$ & $\begin{array}{c}\text { Somewhat } \\
\text { satisfied }\end{array}$ & $\begin{array}{c}\text { Extremely } \\
\text { satisfied }\end{array}$ \\
\hline $\begin{array}{l}\text { 1. How satisfied are } \\
\text { you with your } \\
\text { marriage? }\end{array}$ & 0 & 0 & 0 & 0 & 0 \\
$\begin{array}{l}\text { 2. How satisfied are } \\
\text { you with your } \\
\text { husband/wife as a } \\
\text { spouse? }\end{array}$ & 0 & 0 & 0 & 0 & \\
$\begin{array}{l}3 . \text { How satisfied are } \\
\text { you with your } \\
\text { relationship with your } \\
\text { spouse? }\end{array}$ & 0 & & & & \\
$\begin{array}{l}\text { 4. How satisfied are } \\
\text { you with your sex life? }\end{array}$ & 0 & 0 & 0 & 0 & \\
\end{tabular}

The following are statements about your relationship instability in a general sense. Many marriages go through some ups and downs from time to time. Even people who get along quite well with their spouse sometimes wonder whether their marriage is working out. Please answer the questions with your current marriage in mind.

Yes No

1. Have you ever thought your marriage might be in trouble?

2. Has the thought of getting a divorce or separation crossed your mind?

3. Have you or your husband/wife ever seriously suggested the idea of divorce?

4. Have you discussed divorce or separation with a close friend?

5. Did you talk about consulting an attorney with your spouse? 
Please indicate how you think your current experiences in your marriage compare with your expectations. Here, the expectations are your unique opinions about what is acceptable to you in a marriage. Thus, these expectations are what you realistically expect from your marriage rather than your ideals.

With zero (0) representing your expectation level, please indicate to what degree your marriage currently compares favorably or unfavorably to your expectation level by circling the appropriate number. If your partner or your marital relationship exactly meets your expectation, then you would select 0 .

For example, if you experience more love from your spouse than you would expect, then your answer would be either 1,2, or 3 depending on the degree to which the love you experience is greater than your expectations. A score of 3 means that your experiences of love are much greater than you expect. A score of 1 or 2 would be indicative of your experiences being somewhat greater than you expect. However, your answer would be -3 , if your experiences with your partner was much worse than you would expect.

As another example, if you experience more conflicts in a certain area than you would expect, then your answer would be either $-1,-2$, or -3 . However, if you have less conflicts than you would expect, your answer would be either 1,2, or 3, depending on your expectation level. So please keep in mind that a higher score would indicate a more favorable evaluation of your marriage relative to expectations.

Please indicate how you think your current experiences in your marriage compare with your expectations.

\begin{tabular}{|c|c|c|c|c|c|c|c|}
\hline & $\begin{array}{l}-3 \\
\text { (much } \\
\text { worse } \\
\text { than I } \\
\text { expect) }\end{array}$ & -2 & -1 & $\begin{array}{l}0 \\
\text { (Equals } \\
\text { my } \\
\text { expecta } \\
\text { tion) }\end{array}$ & 1 & 2 & $\begin{array}{l}3 \\
\text { (much } \\
\text { better } \\
\text { than I } \\
\text { expect) }\end{array}$ \\
\hline $\begin{array}{l}\text { 1. The amount of love you get } \\
\text { from your spouse }\end{array}$ & ○ & ○ & ○ & O & 0 & 0 & O \\
\hline $\begin{array}{l}\text { 2. The amount of compatibility } \\
\text { that you experience }\end{array}$ & 0 & 0 & 0 & 0 & 0 & 0 & 0 \\
\hline $\begin{array}{l}\text { 3. The amount of mutual respect } \\
\text { you experience }\end{array}$ & O & 0 & 0 & 0 & 0 & 0 & 0 \\
\hline $\begin{array}{l}\text { 4. The degree to which your } \\
\text { emotional needs are met by your } \\
\text { spouse }\end{array}$ & 0 & 0 & 0 & 0 & 0 & 0 & 0 \\
\hline $\begin{array}{l}\text { 5. The degree to which your } \\
\text { sexual needs are met by your } \\
\text { spouse }\end{array}$ & 0 & 0 & 0 & 0 & 0 & 0 & 0 \\
\hline $\begin{array}{l}6 . \text { The degree to which your } \\
\text { financial needs are met by your } \\
\text { relationship }\end{array}$ & 0 & 0 & 0 & 0 & 0 & 0 & 0 \\
\hline
\end{tabular}


7. The amount of affection your spouse displays

8. The amount of commitment you experience from your spouse

9. The amount your spouse is willing to listen to you

10. The degree to which your interpersonal communications are effective

11. The amount of companionship you experience

12. The amount of relationship equality you experience

13. The amount of confiding that occurs between you and your spouse

14. The amount your spouse is trusting of you

15. The fairness with which money is spent

16. The amount of time you spend together

17. The degree of physical attractiveness of your spouse

18. The amount of conflict over daily decisions that exist

19. The amount of interest in sex your spouse expresses

20. The amount of arguing over petty issues that you experience

21 . The amount of sexual activity that you experience

22. The amount of conflict over the use of leisure time that you experience

23. The amount of criticism your spouse expresses 
24. The amount that you and your spouse discuss sex

25 . The amount to which you and your spouse agree on your lifestyle

26. The amount of disagreement over friends you experience

27. The amount of freedom you experience in pursuing other friendships

28 . The amount to which your spouse supports your choice of an occupation

29. The degree to which the responsibility for household tasks is shared

30. The amount of conflict over money you experience

31 . The amount of jealousy your spouse expresses

32. The amount of privacy you experience

33. The degree to which you and your spouse agree on the number of children to have

34. The amount of responsibility your spouse accepts for household tasks

35 . The amount of conflict over issues involving religious activity

36. The amount of contact that you have with in-laws

37. The amount of conflict you experience with your spouse over issues involving your inlaws

38. The amount of conflict you experience with your spouse over issues involving your parents 
39. The degree of financial support you receive from your parents

40. The degree of financial support you receive from your in-laws

41. The degree of financial support you provide to your parents

42. The degree of financial support you provide to your inlaws

43. The degree to which your spouse's work-related stress affects your relationship

44. The amount of time your spouse spends on electronic devices (e.g., smartphone, tablet PC, computer/laptop, TV, Play Station, etc)

45. The amount of time your spouse spends on electronic devices (e.g., smartphone, tablet PC, computer/laptop, TV, Play Station, etc) when you both are supposed to being doing things together

46. The amount of time your spouse spends working

Thank you for your patience. You are almost finished with this survey. Please answer the following demographic questions in order to provide a full account of who has responded to this survey.

Please indicate your gender.

○ Male

○ Female

Please write your age. 
Please indicate your race.

$\circ$ White

- Black or African American

o Hispanic

- American Indian or Alaska Native

$\circ$ Asian

○ Native Hawaiian or Pacific Islander

○ Biracial/multiethnic

$\circ$ Other

Please select your highest education achieved.

$\circ$ Less than high school

○ High school graduate

o Some college (1-4 years, no degree)

$\circ$ 2-year degree

○ 4-year degree

○ Professional degree (MD, JD, etc)

○ Master's degree (MA, MS, MSW, etc)

○ Doctorate

Please select your occupational status.

○ Employed full time (work 40 hours or more than 40 hours per week)

- Employed part time (work less than 40 hours per week)

○ Unemployed looking for work

$\circ$ Unemployed not looking for work

○ Retired

o Student

o Other 
Please select your partner's occupational status.

- Employed full time (work 40 hours or more than 40 hours per week)

○ Employed part time (work less than 40 hours per week)

○ Unemployed looking for work

○ Unemployed not looking for work

○ Retired

o Student

$\circ$ Other

Please select your annual income.

○ Less than $\$ 20,000$

○ $\$ 20,000$ - $\$ 35,000$

○ $\$ 35,000$ - $\$ 50,000$

○ $\$ 50,000$ - $\$ 75,000$

○ $\$ 75,000$ - $\$ 100,000$

- More than $\$ 100,000$

Please select your partner's annual income.

$\circ$ Less than $\$ 20,000$

$\circ \$ 20,000-\$ 35,000$

$\circ \$ 35,000$ - $\$ 50,000$

$\circ \$ 50,000$ - $\$ 75,000$

$\circ \$ 75,000-\$ 100,000$

$\circ$ More than $\$ 100,000$

How satisfied are you generally with your household's financial situation?

$\circ$ Extremely dissatisfied

○ Somewhat dissatisfied

$\circ$ Neither satisfied nor dissatisfied

○ Somewhat satisfied

$\circ$ Extremely satisfied

How long have you and your partner been in your current marital relationship with one another? (please write your answer in years) 
Are you raising a child/children?
$\circ$ Yes
$\circ$ No

Please list their ages.

$\circ$ The oldest child age

$\circ$ The youngest child age

How many children do you have currently living in your household?
$\circ 1$
$\circ 2$
$\circ 3$
$\circ 4$
o More than 5

Do you currently live with anyone other than members of your nuclear family?
$\circ$ Yes
$\circ$ No

Please indicate them.
$\circ \operatorname{Parent}(\mathrm{s})$
o Parent(s)-in-law
o Siblings or relatives
○ Housekeeper or nanny
$\circ$ Others 